\title{
L'intervento in Psicologia Clinica Perinatale. Integrazione con i percorsi di cura in ostetricia, neonatologia, pediatria, neuropsichiatria infantile
}

\subsection{Le disfunzioni precoci nella relazione genitore-bambino}

I genitori e la famiglia, soprattutto nei primi anni di vita del figlio, svolgono una funzione fondamentale di matrice del suo sviluppo neuromentale affettivo-cognitivo e sociale (Imbasciati, 2006a). Quando qualcosa nella relazione primaria genitore-bambino non funziona ottimamente, per la struttura psichica del genitore, per avvenimenti traumatici, problemi riferibili all'ambiente esterno, disagi psicosociali o conflittualità familiari (depressione post partum, psicosi puerperali), anomalie sopraggiunte durante la gravidanza o il parto (gestosi o altre patologie, nascita prematura, gemellare, patologie del bambino) oppure a seguito di maltrattamenti, abusi, abbandoni subiti da parte del proprio genitore, tutte le situazioni a rischio nell'ambito della genitorialità in cui le funzioni di cura e protezione dei figli sono disturbate e influenzano la qualità della relazione genitore-bambino (Candelori, Mancone, 2001a) - i sintomi di malessere nei neonati e nei bambini piccoli si manifestano psicosomaticamente attraverso i canali della comunicazione corporea. Nel neonato la sofferenza si esprime attraverso il corpo, con sintomi fisici, così come tutta la comunicazione bambino-caregiver avviene attraverso i canali di una comunicazione corporea. Crescendo, il bimbo potrà disporre di mezzi più elaborati per esprimere il suo stato di sofferenza psicologica, attraverso i disturbi del comportamento. Molteplici e complessi si presentano i quadri clinici con cui si esprime la psicopatologia infantile nel corso dello sviluppo del bambino, dall'infanzia fino alla adolescenza: importanti manuali di psicopatologia dell'infanzia (Ammaniti, 2001a) evidenziano in particolare i disturbi della regolazione fisica ed emotiva (Speranza, 2001a), dell'alimentazione

L. Cena $(\varangle)$

Professore Associato di Psicologia Clinica

Dipartimento Materno Infantile, Facoltà di Medicina e Chirurgia

Università degli Studi di Brescia

cena@med.unibs.it 
(Lucarelli, 2001), del sonno (Lucarelli, Vismara, 2001), delle emozioni (Fabrizi, 2001), i disturbi post traumatici da stress, nonché gli effetti di abusi (Ammaniti, 2001b), i deficit dell'attenzione/iperattività e disturbi della condotta (Speranza, 2001b), i disturbi multisistemici dello sviluppo (Muratori, Cosenza, Parrini, 2001), dell'identità di genere (Coates, Cook, 2001).

Ammaniti segnala la diversità dei differenti sistemi di classificazione diagnostica, con una tassonomia psicopatologica, operata attraverso un Manuale diagnostico e statistico dei disturbi mentali, il DSM-IV (American Psychiatric Association, 1994), sia con la Classificazione delle sindromi e dei disturbi psichici comportamentali, ICD 10 (World Health Organization, 1992), sia ancora e poi con il PDM, Manuale Diagnostico Psicodinamico (2006). Il PDM si discosta in modo significativo dal DSM-IV e dall'ICD in quanto fornisce un nuovo approccio alla diagnosi: per il DSM-IV e l'ICD la classificazione diagnostica è identificata con modalità categoriali, mentre nel PDM assume un senso dinamico e dimensionale. Questo diverso orientamento sposta la considerazione a una prospettiva più ampia sulla singolarità dell'individuo, con un'attenzione non solo alla psicopatologia, ma anche alle risorse del soggetto. La salute mentale viene descritta nel PDM, secondo quanto prospettato nel modello biopsicosociale (Engel, 1977), come qualcosa di più dell'assenza di sintomi psicopatologici, come presenza e adeguatezza di tutte le funzioni del soggetto, cognitive, affettivo emotive e comportamentali e da una condizione di benessere nell'ambito del contesto in cui vive l'individuo. La definizione dei disturbi psichici nell'infanzia è dunque controversa. Alcuni quesiti che vengono sollevati (Ammaniti, 2001c) sono in primo luogo che la psicopatologia infantile, rilevabile in un funzionamento o comportamento anomalo del bimbo non può essere inquadrata come un disturbo individuale, al pari di quelle dell'adulto, ma vada considerata essenzialmente all'interno dell'ambiente relazionale entro cui il minore sta vivendo; in secondo luogo, sarebbe aleatorio rilevare la psicopatologia infantile nei primi anni di vita, quando il bimbo non sa ancora parlare ed esprimere i suoi disagi; infine, è dubbio circoscrivere come patologiche le manifestazioni della prima infanzia, periodo in cui avvengono repentini cambiamenti nel funzionamento individuale e dell'ambiente relazionale del bimbo. In sostanza, non sarebbe possibile dirimere se i problemi che insorgono nella prima infanzia siano dei segnali di quadri psicopatologici che si manifesteranno più in là nello sviluppo.

Per quanto riguarda la diagnosi in età infantile (Ammaniti, 2001d), il sistema di classificazione attualmente più in uso è la Classificazione Diagnostica 0-3, del National Center for Clinical Infant Program (Zero-to-Three, 1994) di Washington, che consente di prendere in considerazione la qualità della relazione genitore-bambino: il disturbo del bimbo viene collocato entro il contesto relazionale genitore-bambino. In questa Classificazione l'aspetto relazionale genitore-bambino viene considerato in un apposito asse e viene presa in considerazione una valutazione del bambino che implica la valutazione della qualità della relazione coi genitori e con l'ambiente. Ciò che invece sembra ignorato da tutti questi sistemi di classificazione diagnostica (DSM-IV, ICD 10, Classificazione Diagnostica 0-3) è la clinica psicosomatica, prospettiva notevole per lo studio di certe manifestazioni infantili (Candelori, Mancone, 2001b). 
Un approccio alla clinica psicosomatica nell'infanzia implica connotazioni ancora più complessificate, sia per l'overlapping di discipline diverse che si occupano degli ambiti corporei e psichici del bambino, sia anche per la presenza di quelle dinamiche proprie dei processi di cambiamento che caratterizzano l'età evolutiva. Le complessità sono rilevanti per la valutazione diagnostica. La difficoltà di un'elaborazione nosografica dei disturbi psicosomatici è del resto desumibile dalla loro diversa collocazione a seconda dei sistemi di classificazione. La classificazione nosografica utilizzata dalla psichiatria (DSM-IV) indica per esempio "somatizzazione", invece di disturbo psicosomatico: nella prima infanzia una "somatizzazione" si manifesta infatti sotto forma di disturbi ricorrenti che possono rientrare nella normalità, mentre i criteri per una valutazione di psicopatologia (disturbo o "malattia"), così come invece è identificata per gli adulti, è quanto mai inadeguata.

In tutta la prima infanzia la manifestazione del disagio avviene prevalentemente attraverso espressioni somatiche che pertanto andrebbero considerate come normale mezzo di comunicazione anziché come disturbo. Molte di queste manifestazioni sono specifiche: si tratta di canali mediante i quali il bambino richiama attenzione (affetto), comunicando un disagio di natura emotiva che non è ancora in grado di elaborare e di esprimere altrimenti. Secondo la terminologia pediatrica in uso, i disturbi sono classificati a seconda dell'impatto sulla funzione interessata: sonno, alimentazione, escrezione, respirazione, motricità. La nosografia in uso codifica i sintomi attraverso raggruppamenti in sindromi identificate principalmente attraverso criteri medici. La prospettiva psicosomatica attuale integra del resto i sintomi anche per gli adulti in un equilibrio mentale e relazionale (Kreisler, 1981). Coliche dei primi mesi, dermatiti, eczemi, dolori addominali sono alcuni dei più frequenti messaggi che il corpo del neonato e del bimbo molto piccolo comunica a chi si prende cura di lui. Quello psicosomatico è un malessere che egli sperimenta realmente sul proprio corpo, quale unica via attraverso la quale si esprimono quei disagi di cui egli non è e non può essere consapevole.

Anche le sindromi psicosomatiche riscontrabili nell'adulto (Baldoni, 2010) riflettono analoga possibilità di esprimere il disturbo psichico a livello somatico: questi adulti infatti hanno spesso scarse capacità strutturali di elaborare ed esprimere le proprie emozioni, presentando un'insufficienza dei "processi di mentalizzazione", mancanza di una capacità fantasmatica, mentre si mantiene intatta la capacità di funzionamento cognitivo, orientato agli aspetti concreti del mondo esterno. Si tratta del cosiddetto "pensiero operatorio" (Marty, de M'uzan, 1963): i vissuti conflittuali non possono acquisire consapevolezza; è quest'individuo l'alessitimico che non riesce né a "sentire" né tantomeno a comunicare i propri stati emotivi (Imbasciati, Margiotta 2008b, capitolo 8).

Dagli studi sviluppati dall'Infant Research il costrutto dell'alessitimia è stato del resto integrato con quello della Regolazione Affettiva (Taylor et al., 2000), verso un unico modello che possa chiarire l'influenza delle emozioni sulle funzioni corporee, così come si registra nell'ambito delle interazioni genitore-bambino. Il bambino quanto più è piccolo tanto più è "psicosomatico": la mente prende origine dal corpo e il corpo del bambino, in età prelinguistica, rappresenta il mezzo per comunicare ai genitori o ad altri caregiver significativi la propria condizione. Nella prima infanzia il soma e la mente si trovano in uno stato di indifferenziazione: la mente scaturisce 
dal corpo (Solano, 2001), lo sviluppo della mente (Imbasciati, 2006a) avviene attraverso un processo graduale a partire dal corpo (Imbasciati, 2008a). Corpo e mente sono considerati come un continuum funzionale in cui si svolge il processo di differenziazione delle funzioni mentali. Il disturbo psicosomatico documenta nell'adulto un difettoso funzionamento della mente rispetto alla sua differenziazione dal corpo, mentre il bambino esprime col corpo il disagio. Problemi nella funzione di regolazione del genitore nelle "interazioni emozionali" possono avere una costante ricaduta nella formazione della capacità del bimbo di rappresentazione mentale delle esperienze che attraversa. Se i disturbi della regolazione emozionale genitore-bambino sono oltre una certa misura, questo bimbo può essere considerato a rischio di sindromi psicosomatiche nell'età adulta.

Nella letteratura pediatrica i sintomi somatici non interpretabili hanno ricevuto scarsa considerazione, cosicché il problema di una diagnosi di disturbo da "somatizzazione", come potrebbe essere possibile per l'età adulta (DSM-IV), prima della fase adolescenziale è molto controversa (Cerutti, Guidetti, 2007). Le indicazioni circa l'epidemiologia dei disturbi somatoformi nella prima infanzia, così come quelle per le modalità di trattamento, sono poche: lo studio della psicopatologia nell'infanzia dovrebbe tenere in considerazione molteplici costrutti (multiple construct) tra di loro diversi a secondo delle Scuole, attraverso osservazioni cliniche in più contesti ( $\mathrm{mul}$ tiple contest) condotte da vertici differenti, da più informatori (multiple informants), e con metodi diversi (multiple method) (Presaghi, Cerutti, 2007).

I sintomi alla consultazione pediatrica, nella prima infanzia, possono presentarsi con determinate caratteristiche a seconda dell'età del bambino e dello sviluppo dei vari organi corporei, dove interessano soprattutto le funzioni digestive (coliche dei primi mesi, vomito o inappetenza, mal di pancia), respiratorie (asma) o la pelle (dermatiti, eczemi): più che il tipo di sintomo, il particolare evento familiare che può averlo preceduto (nascita di un fratellino, un lutto in famiglia o l'ingresso a scuola), collegato al particolare contesto delle relazioni familiari in cui esso si è manifestato, permetterà di comprendere il significato del disturbo e i particolari bisogni espressi in tal modo. La sofferenza deve essere letta e decodificata in relazione ai suoi contesti di appartenenza, in primo luogo quello relazionale familiare. L'osservazione del bambino nel contesto di sviluppo (Cammarella, Lucarelli, Vismara, 2001), effettuata con le figure affettive di riferimento (Imbasciati, Cena, 2010), è la metodologia privilegiata, che ci fornisce dati diretti sull'espressione dei disturbi del bimbo e che, integrata con altre rilevazioni riportate dai genitori e dagli adulti che si prendono cura di lui, consente di delineare un prospetto valutativo della psicopatologia infantile.

Disturbi psicosomatici si possono manifestare in tre periodi significativi della prima infanzia: in una fase primaria già alla nascita, o poco dopo, nel primo trimestre; dal secondo semestre all'età di quindici-diciotto mesi, periodo costitutivo dell'attaccamento; e nella fase ultima della prima infanzia, dalla metà del secondo anno alla metà del terzo. Ogni fase può presentare disturbi ricorrenti: la colica cosiddetta idiopatica si manifesta in genere verso il dodicesimo-quindicesimo giorno, quando il lattante stabilisce le prime relazioni; l'insonnia può comparire precocemente, nel primo trimestre di vita, ma anche più tardi, nel secondo e terzo anno; disturbi alimentari possono presentarsi nel secondo semestre, con il passaggio allo svezzamento; il 
singhiozzo spastico è disturbo prevalente nel secondo e terzo anno.

Negli attuali sviluppi della psicosomatica francese il corpo del neonato viene considerato come equivalente a una scena psichica (Golse, 2008): l'attenzione al corpo del bebè è un elemento fondamentale all'interno delle consultazioni terapeutiche perinatali. Non può esistere una psicoanalisi della prima infanzia senza un'implicita valutazione della situazione psicofisica del corpo: l'esame del funzionamento corporeo occupa una posizione centrale in ogni terapia perinatale, così come il neonato non può essere concepito al di fuori delle sue relazioni (Golse, 2008). La clinica del bebè non può essere pertanto descrittiva e centrata solo sul bimbo: sono gli aspetti interattivi che consentono al clinico di percepire, controtransferalmente, i fenomeni psichici in termini di affetti, emozioni, stati, strutture verso cui possono essere rivolte le strategie terapeutiche.

Anche da un altro vertice di lettura, quello della terapia sistemica, gli studi di Minuchin (Kowallis, 1995) mettono in evidenza il grado di influenza delle modalità relazionali dei genitori sulla patologia psicosomatica del bambino.

Gli atteggiamenti iperprotettivi dei genitori, piuttosto che favorire un possibile miglioramento del disturbo, aprono la strada a circoli viziosi, che incrementano le probabilità di cronicizzazione della patologia. Le modalità interpersonali dei genitori, inoltre, giocano un ruolo importante nella percezione dell'autostima del bambino malato, nel rapporto con i fratelli e nella relazione fra i coniugi.

In letteratura sono stati soprattutto gli studi di Kreisler (Kreisler, 1981; Kreisler et al., 1974), a partire da quelli di Spitz (1946) e di Winnicott (1958), che hanno messo in evidenza come le psicopatologie del lattante vadano affrontate secondo una prospettiva psicosomatica in chiave relazionale. La multipla causalità relazionale è tanto più evidente quanto più un bimbo è lattante, durante il primo anno di vita: non facile è però individuare nella rete relazionale i punti focali che hanno agito disturbando il bimbo. Qui il compito del pediatra, alle cui cure è portato il bimbo in prima istanza, necessita di una competenza non facile da acquisire, nonché di pazienza e disponibilità di tempo non certo favoriti dall'attuale assetto dei servizi sanitari. Auspicabile sarebbe un lavoro in equipe integrata (pediatra, neonatologo, psicologi clinici esperti in perinatalità, neuropsichiatri infantili, assistenti sociali) entro il quale i genitori potessero trovare accoglienza adeguata e diventare terapeuti integrati nell'equipe. Nella maggioranza dei casi, quanto più i bimbi sono piccoli, tanto più i genitori /caregiver devono essere il fulcro su cui mirare l'azione terapeutica. Problematiche di regolazione dei ritmi del sonno, alimentazione, infezioni, disturbi intestinali, dermatologici ed altri, quando non sono riscontrabili evidenze di disfunzioni organiche, possono essere considerati espressioni somatiche di disturbi psichici relazionali: fuorviante è pertanto considerare "paziente" il piccolo e concentrare le cure su di lui; occorre curare l'insieme relazionale in cui egli si trova, genitori in primo luogo.

Kreisler (1985) individua nell'eziopatogenesi dei disturbi dell'età infantile due tipologie di fattori ambientali: il sovraccarico delle eccitazioni e l'insufficienza o carenza delle stimolazioni; entrambi questi ordini di fattori hanno un fulcro nel tipo delle cure genitoriali. Non facile è però, nell'uno e nell'altro caso, individuare più precisi momenti patogeni. 
Nei primi mesi di vita l'assetto psicosomatico è mantenuto attraverso il funzionamento interattivo con i genitori. In un ambiente familiare patogeno i genitori, spesso a loro volta vittime di rifiuto o frustrazione da parte dei propri genitori, tendono a riprodurre le stesse modalità relazionali nei riguardi del loro bambino, con una ripetizione transgenerazionale. Sentimenti genitoriali ambigui, fra il bisogno di inglobare il bambino e la tendenza all'abbandono, possono condurre a interazioni dominate da un'alternanza disorganizzante tra vuoto relazionale ed eccitamento disordinato (Kreisler, 1992). Tra i disturbi comportamentali più frequenti nel primo semestre di vita viene osservata una povertà di comunicazione e un evitamento dello sguardo, indici semiologici, secondo Kreisler, dell'insufficienza primaria dell'attaccamento (Kreisler, 1992). Nel secondo semestre e nel secondo anno le sintomatologie psicosomatiche più frequenti possono implicare la compromissione di molti settori dello sviluppo, come l'espressione psicomotoria e il linguaggio; anche l'acquisizione dello schema corporeo può presentare anomalie e difetti. Alla fine del secondo anno, se le patologie relazionali permangono, il quadro clinico può diventare ancora più alterato, in una disorganizzazione strutturale. Eventi traumatici e comunque situazioni conflittuali possono condurre a una disorganizzazione psicosomatica: l'importanza dell'evento traumatico non dipende tanto dalle sue caratteristiche intrinseche quanto soprattutto dalla risonanza relazionale multipla che viene elaborata nel bimbo. È comunque evidente l'importanza della qualità della primaria relazione genitore-bambino nella determinazione dello stato di salute del neonato: il caregiver può non funzionare adeguatamente e non proteggere il bimbo, o non fornirgli un sostegno adeguato esponendolo a stimolazioni eccessive, oppure non sollecitarlo adeguatamente, oppure ancora esponendolo a una discontinuità sfavorevole a un armonico sviluppo (Kreisler, 1992; Kreisler e Cramer 1981). Le osservazioni sulle prime interazioni madre-neonato hanno evidenziato che sin dall'inizio la madre mette in gioco con il suo bimbo i conflitti e le angosce che avevano fondato la sua relazione primaria. Cramer e Stern (1988) attraverso l'uso di videoregistrazioni nel primo anno di vita hanno evidenziato come l'effetto patogeno di vissuti rimossi possano essere agiti dalla madre nell'interazione precoce con il bimbo.

Gaddini denomina le malattie psicosomatiche della prima infanzia "sindromi psicofisiche" (1981): quella più precoce secondo l'autore risulta essere il mericismo, che compare intorno alle otto settimane di vita; la dermatite atopica interviene intorno al sesto mese di vita, l'asma bronchiale dopo il primo anno di vita; la balbuzie è una sindrome psicofisica che si può manifestare nel secondo anno di vita, qualora non si sia instaurata l'asma (Gaddini, 1982). L'autore considera l'esordio precoce come un segno di maggiore gravità in quanto si assiste nell'interazione primaria a una precocità del processo di separazione, che di per sé può essere traumatico: lo sviluppo della mente è un processo graduale nella direzione dal corpo alla mente, una sorta di emergenza dal corpo, che coincide con la graduale acquisizione mentale del Sé corporeo e tale processo di emergenza non può prescindere dalla funzionalità unita del bambino con la sua mamma (Gaddini, 1982).

Un'adeguata relazione genitore-bambino è pertanto indispensabile per il conseguimento di un sano sviluppo, nel raggiungimento di un valido equilibrio psicosomatico. Anche Winnicott (1965) individua nell'ambiente un fattore fondamentale af- 
finché il processo maturativo possa procedere regolarmente. Attraverso la descrizione dei fenomeni transizionali l'autore valorizza l'importanza della madre nella regolazione del processo di crescita (Winnicott, 1958; 1971) e come la non presenza di fenomeni transizionali possa essere un segnale di un eventuale processo di disequilibrio psicosomatico. Il bambino nasce con una capacità di autoregolazione comportamentale, ma questa può essere sviluppata solo attraverso la rilevanza della figura materna, quale insostituibile regolatore dei comportamenti e delle diverse funzioni fisiologiche del bambino (Taylor, 1987): emblematiche sono a tale proposito le ricerche sperimentali di Hofer (1984). Oltre ai comportamenti di reciproca regolazione osservabili nella relazione madre-bambino, ci sono anche altri processi nascosti, per cui la madre "svolge la funzione di regolatore biologico e comportamentale per il bambino" (Hofer, 1983a,b).

La relazione genitore-bambino costituisce un sistema di regolazione che dipende dalla capacità del bambino di emettere segnali congrui con i suoi bisogni e questa capacità dipende dalla sensibilità e dall'attenzione della madre a questi segnali e nell'insegnare al bimbo a usarli, rispondendo a sua volta ai congrui segnali della madre, in modo da creare un codice di comunicazione condiviso. Le difficoltà possono nascere nel bambino, ma sono originate da un difetto della capacità della madre e possono culminare in una disritmia o in una discordanza dei loro scambi regolatori (Grotstein, 1986) che si manifesta come patologia nel corpo, soprattutto se il bimbo è piccolo, e poi nel comportamento e quindi nella mente.

\subsection{La mente che nasce dal corpo}

L'Infant Research ha studiato per via sperimentale gli scambi comunicativi primari nella relazione madre-bambino a partire dalle prime forme di imitazione e ha individuato nella madre le competenze di regolazione delle emozioni del bimbo, sia come etero-regolazione che come autoregolazione. Una buona regolazione viene identificata come la capacità del bimbo di mantenere la propria organizzazione comportamentale rispetto a stati di tensione e stress emotivo: egli apprende questa funzione nell'ambito delle interazioni primarie con la madre, la quale progressivamente gli insegna a organizzare e regolare i suoi stati (Sander, 1987) in un sistema interattivo: è quella che viene definita intersoggettività primaria (Trevarthen, 1980). Questo sistema di regolazione ha origine a un livello di organizzazione biologico-neurofisiologico-comportamentale e progressivamente evolve nella capacità di formare simboli, pensare e usare il linguaggio (Sander, 1975; Lichtenberg, 1983).

Gli stati comportamentali e fisiologici del bambino sono caratterizzati da periodicità e ritmicità e cambiano con la sollecitazione da parte della madre. Oltre ai macroritmi, che sono implicati nell'allattamento, nel sonno, nell'evacuazione e nel ciclo fondamentale quiete-attività, ci sono dei microritmi implicati nella suzione, nel pianto e in certi comportamenti stereotipati (Emde, Robinson, 1979). Tra madre e bambino si stabilisce una sincronia di fasi, il cui livello dipende dal progressivo reciproco adattamento segnalato dalle interazioni, in cui il comportamento di ciascun indi- 
viduo influenza quello dell'altro e ne è a sua volta influenzato. Studi condotti da Brazelton (1973) mettono in evidenza come già a due o tre settimane di vita i bambini rispondono con modelli di attenzione, azione e affettività, qualitativamente diversi.

Stern rileva come il neonato, fin dalla seconda settimana, abbia la capacità di ricercare e regolare gli stimoli in entrata, impegnandosi nell'interazione con la madre, discriminandola e riconoscendola (Stern, 1977; Emde, Robinson, 1979). La sensibilità nel neonato alla stimolazione è molto bassa nelle prime settimane di vita e progressivamente aumenta con la crescita: il neonato risponde a livelli ottimali di stimolazione al di sotto dei quali la stimolazione è ricercata e al di sopra dei quali la stimolazione è evitata (Stern, 1983). Gli studi sulla sintonizzazione affettiva di Stern (1985) secondo cui la madre, a partire dal secondo mese di vita, attraverso imitazioni delle espressioni del proprio bimbo riesce a sintonizzarsi rimandandogli emozioni e affetti in forme diverse, hanno messo in evidenza come per lo sviluppo delle strutture mentali sia importante la creazione di un dialogo non verbale sintonico. Stern (1977) descrive una madre e un bambino che creano cicli periodici di attività sincronica e sintonica, più simili alla relazione tra i partner di una danza che tra antagonisti di una partita di tennis, in una sorta di creazione virtuosa, piuttosto che altrimenti sconnessa. In questa reciproca interazione di regolazione ci possono essere slanci troppo lunghi, o troppo brevi, da parte di entrambi i partecipanti che devono quindi accendersi o spegnersi per produrre gli stati sintonici desiderati. Nelle argomentazioni dell'autore ha rilievo il concetto dell'altro regolatore del Sé (1985): il bambino dipende inizialmente da un altro, come "altro regolatore del suo Sé". Il tipo di esperienza di un "essere con" un altro regola il Sé e forma gradualmente delle RIG (rappresentazioni interne generalizzate) di se stesso in interazione con l'altro. Questo concetto consente di capire le modalità di "essere con", di particolare rilevanza quando si tratti di madri i cui bambini hanno disturbi psicosomatici, e quanto esse possano influire sulle funzioni di regolazione del bimbo nei primi mesi di vita.

Tra il settimo e il nono mese di vita i bambini giungono gradualmente a capire che le esperienze della mente sono condivisibili con gli altri: quando il bambino si accorge che gli altri, pur separati e distinti da lui, possono avere e conservare uno stato mentale simile al suo, è in grado di condividere l'esperienza soggettiva con loro. È quella che viene definita intersoggettività secondaria (Trevarthen, Hubley, 1978).

La capacità di riconoscere le proprie emozioni è un fattore determinante nella trasmissione delle prime modalità relazionali e di attaccamento tra genitore e figlio (Bowlby, 1988): i Modelli Operativi Interni, raffrontabili alle RIG di Stern. Tale capacità appare profondamente influenzata dal tipo di accessibilità emotiva che il genitore ha potuto a sua volta sperimentare nei confronti delle proprie figure di attaccamento nel corso della propria storia infantile.

Le modalità relazionali precoci tra il bambino e i suoi caregiver danno origine a stili individuali di regolazione affettiva che si ritrovano alla base dei successivi pattern di interazione: la modalità di regolazione emotiva che si attiva e si modula durante la relazione tra il bambino e il genitore viene interiorizzata e le esperienze affettive precoci influenzano e differenziano l'organizzazione dei legami di attaccamento, considerati relazioni di tipo emozionale attraverso cui il bimbo impara peculiari 
stili di regolazione. Questi vengono considerati strategie adattative e difensive nei confronti della disponibilità emotiva delle figure affettive di riferimento (Main, 1996), che il bambino impara per far fronte alle varie esperienze emotive che gli consentono di conservare il legame con il genitore.

L'attaccamento sicuro nel bimbo è correlato alla sua capacità di regolare il proprio stato emotivo, mentre l'attaccamento insicuro implica disfunzioni nelle capacità regolatorie del bimbo il cui genitore non è disponibile a modulare a sua volta i suoi livelli di attivazione (Cassidy, 1994). Modelli di attaccamento insicuri sono collegati a fallimentari tentativi di regolazione affettiva. La teoria dell'attaccamento ha contribuito notevolmente a mettere in evidenza la caratteristica dei comportamenti non verbali materni negli scambi interattivi e l'importanza della sintonia, nel contatto fisico ed emotivo, nella costruzione dei diversi pattern di attaccamento. La disponibilità emotiva genitoriale a regolare gli stati affettivi del bimbo è fondamentale per il costituirsi del legame di attaccamento: il grado di consapevolezza emotiva materna (Nichols, Gergely, Fonagy, 2001) costituisce la "sensibilità" materna (Ainsworth, 1979; Crittenden, 1979-2004); nei termini di Stern (1985) si tratta di capacità di sintonizzazione che indica quella capacità genitoriale di soddisfare i bisogni emotivi del bimbo con modalità e in tempi adeguati al suo sviluppo: le madri modulano i loro ritmi a quelli di attivazione dei loro bambini e li aiutano a regolare il loro livello di attivazione. La Ainsworth rileva come i pattern di attaccamento sicuri e insicuri che il bimbo manifesta nei confronti del caregiver dipendono dalla sensibilità degli adulti di cui ha potuto fruire nel corso del primo anno di vita.

Le attuali ricerche convergono dunque nel rilevare come lo sviluppo psichico dell'individuo dipenda non tanto da fattori biologici quanto da apprendimenti precoci in epoca fetale e nei primi mesi di vita (Imbasciati, Calorio, 1981; Imbasciati, 2006 b). Le alterazioni biologiche condizionano alcune grosse patologie dello sviluppo psichico, ma non sembrano influire gran che sulla psicopatologia dell'adulto, né tantomeno sulla qualità dello sviluppo psichico entro i limiti della cosiddetta normalità. Tuttavia è proprio tale "qualità", l'efficienza delle funzioni psichiche, che determinano il destino di un uomo e il suo adattamento sociale. La massima attenzione delle ricerche è dunque concentrata su quei fattori, psichici e sociali, che determinano nelle prime epoche di vita, la "costruzione" dell'apparato mentale che caratterizzerà il singolo individuo (Imbasciati, Dabrassi, Cena, 2011). Gli “apprendimenti” precoci, che vengono indicati come le pietre miliari della successiva costruzione, per progressivi apprendimenti, delle varie e progressive funzioni psichiche, dipendono dalla "relazione" che si stabilisce tra il neonato, il feto e la persona (o le persone) che si prendono cura di lui: la madre in primis. Tale relazione è stata studiata dai più recenti sviluppi della ricerca, che vede oggi coniugate fecondamente le osservazioni cliniche della psicoanalisi infantile e le metodologie sperimentali sui primi mesi di vita. Anche l'equilibrio psicosomatico, nella sua ottimalità o in eventuali patologie, dipende dai fattori relazionali della prima infanzia. Di qui l'importanza di simili studi sull'argomento: sono in gioco le condizioni che determinano la "qualità" della salute, fisica e psichica, delle future generazioni. La qualità della "relazione" è fondata sulla qualità e l'efficacia della comunicazione che si stabilisce tra il genitore $\mathrm{e}$ il bimbo: questa è mediata quasi totalmente da canali non verbali e la sua elabora- 
zione avviene prevalentemente a livello non consapevole; di qui la complessità dei rilievi sperimentali che possano fornire dati utili per un intervento efficace sulla relazione. La Psicologia Clinica Perinatale ha messo in evidenza come per comprendere i meccanismi psichici che più contribuiscono alle patologie psicosomatiche sia necessaria una valutazione attenta che consenta di comprendere la qualità delle interazioni. Oltre a precisare le caratteristiche del bambino e le modalità del suo funzionamento psichico, è indispensabile valutare la personalità della figura chiave della relazione (che di solito è la madre) soprattutto nel suo funzionamento nel ruolo materno. Opportuno è valutare anche la personalità del padre, del contesto familiare e sociale, nonché individuare gli eventi scatenanti gli eventuali disturbi osservati in rapporto all'evoluzione del bambino e alla sua biografia, interpretando le circostanze sfavorevoli nelle dinamiche dell'interazione.

\subsection{Sviluppi applicativi della Teoria dell'Attaccamento}

Nella succitata prospettiva si collocano i nuovi sviluppi evoluzionistici della teoria dell'attaccamento: Patricia Crittenden fondatrice della IASA (International Association for the study of Attachment) con diversi clinici internazionali sta sviluppando interessanti ricerche sulla psicosomatica. Secondo la Crittenden i "disturbi psicosomatici" si presentano come problemi: in cui il corpo sembra sapere ciò che la mente non può dire.

Alle informazioni e alle rappresentazioni "cognitive" e "affettive" del suo Modello Dinamico-Maturativo (capitolo 7) sono state aggiunte in questi ultimi anni dall'autrice informazioni e rappresentazioni "somatiche": la Crittenden tuttavia sostiene che la nostra prima informazione, nella vita, è somatica ed è la più importante per la sopravvivenza. Tante sono le informazioni rappresentate somaticamente, e lo psicoterapeuta deve prenderle in attenta considerazione (Crittenden, 2011). La Crittenden evidenzia come non accada nulla nel comportamento umano o nella mente senza una partecipazione del corpo e fa riferimento alle neuroscienze con le ricerche di Botvinik e Eisenberg (Botvinik et al., 2005; Eisenberg et al., 2003): quando percepiamo dolore, sia a livello fisico che psichico, il cervello registra le medesime rappresentazioni sia nelle aree specifiche della localizzazione percettiva, sia nella corteccia cingolata anteriore, ovvero in un' area che interverrà nell'elaborazione emotiva e nella regolazione somatica. Un intervento orientato alla salute e al benessere psicofisico dell'individuo deve pertanto tenere presente il corpo, oltre che la mente e tutto il comportamento: il comportamento stesso è psicosomatico (Crittenden, 2011).

Tra le numerose ricerche condotte dai clinici della IASA, periodicamente pubblicate sulla rivista internazionale DMM News, alcune ricerche recenti stanno portando evidenze cliniche in relazione alla ricerca sul legame tra lo stress durante l'accudimento precoce infantile e la qualità della relazione con il genitore come predittori degli effetti sulla salute mentale e fisica del bambino. Tali ricerche (Kaplan et al., 2010) evidenziano come lo stress quotidiano, quando è troppo elevato o si protrae per lunghi periodi di tempo, può causare cambiamenti che influenzano lo sviluppo 
psicosomatico dell'individuo. La salute del neonato sottoposto a stati di stress elevato è a rischio: quando i genitori manifestano disturbi mentali, dipendenze, comportamenti violenti o comunque modalità di cura imprevedibili per il bimbo, trascuranti o abusanti lo espongono a stati prolungati di stress. Il sistema di regolazione dello stress può restare nel bimbo costantemente regolato a un livello elevato e questa sovraeccitazione può portare all'espressione di disturbi psicosomatici, per esempio asma e disturbi respiratori (Costa-Pinto et al., 2010; Kozyrskyj et al., 2008), spesso con cambiamenti stabili della struttura e della funzione di alcune aree cerebrali (Gunnar et al., 2009). I glucocorticoidi (cortisolo) messi in circolo dall'asse ipotalamo-ipofisiario (HPA) aumentano in risposta agli stressor. Quando lo stress è sotto controllo, prevedibile e per brevi periodi, l'asse HPA promuove l'adattamento e la resilienza. Ma quando le situazioni stressanti sono incontrollabili, non prevedibili e pervasive, la stimolazione può diventare "nociva" e alterare il funzionamento stesso dell'asse HPA.

Di solito il cortisolo è secreto con un ritmo diurno, bassi livelli al risveglio, seguiti da un picco a metà mattina e da un graduale declino verso sera. Nelle madri affette da depressione post partum, i neonati hanno spesso un minore declino nel corso della giornata. In altre parole, presentano un pattern differente, caratterizzato da livelli persistentemente elevati di cortisolo per tutto il pomeriggio. I bambini di madri che al mattino rispondono ai bisogni del loro bimbo in modo adeguato e con una comunicazione, verbale e non verbale, sincrona e contingente, consentiranno una riduzione nel pomeriggio dei livelli di cortisolo nel bimbo (Letourneau et al., 2011); ciò conferma nell'uomo un processo che è noto da tempo nei cuccioli di ratto descritti nelle ricerche di Hofer. Le esperienze dirompenti, di abuso, di trascuratezza o imprevedibili inondano il cervello di cortisolo: l'attivazione cronica dell'asse HPA ha conseguenze dannose per lo sviluppo del bambino ed elevate dosi persistenti di cortisolo mettono in pericolo la crescita neuronale e le connessioni dendritiche. In questo modo lo stress nocivo lascia un'impronta biologica di danno persistente sulla struttura e sulla funzione cerebrale (Shonkoff, 2004). Le due principali regioni del cervello che sono colpite dagli stress nocivi sono l'ippocampo e la corteccia prefrontale. Queste regioni sono in relazione con la memoria e l'autoregolazione e costituiscono le basi dello sviluppo cognitivo e socioemotivo. Nel tempo, gli effetti dello stress nocivo sulla struttura e la funzione cerebrale possono modificare i pattern di comportamento da un'organizzazione contesto-sensibile a modelli di organizzazione rigidi e stereotipati. Questi bambini sono meno capaci di risolvere i problemi in modo autonomo.

La relazione tra esposizione alla depressione materna nei primi due anni di vita e iperattività tra i due e gli otto anni di età evidenzia un esito potenziale di esposizione cronica allo stress nocivo (Letourneau et al., 2006); lo stress nocivo può influenzare la risposta infiammatoria (Gunnar et al., 2009) e gli effetti dello stress nocivo sono particolarmente preoccupanti nella prima infanzia perché il cervello, durante questa fase di rapido sviluppo, è estremamente sensibile all'esperienza. Il futuro sviluppo del cervello infantile dipende dallo sviluppo cerebrale attuale e passato. In questo modo, le modifiche della struttura e della funzione cerebrale determinate dallo stress nocivo producono cambiamenti cumulativi che colpiscono non solo gli aspet- 
ti attuali di sviluppo a un dato stadio, ma anche le fasi successive: il cervello si adatta alle circostanze passate e presenti e questo può vincolare gli individui a tali circostanze.

\subsection{Modelli di intervento in Psicologia Clinica Perinatale}

Nel contesto di un trattamento il genitore si rivolge per un problema manifestato dal bambino, ma raramente lo ritiene legato alla relazione che egli ha strutturato con il figlio: questo crea difficoltà, rispetto alle idee tradizionali di terapia. Una radicata, quasi inconsapevole e creduta ovvia stereotipia della nostra tradizione medica, individua come "paziente", da curare quindi, colui che patisca, o comunque manifesti, un inconveniente. Malgrado da parecchie decadi si sia dimostrato che tale assunto è fallace, una radicata idea perdura nella nostra popolazione, non solo in psicopatologia (vedi il concetto di "paziente designato" della Scuola Sistemica) ma anche in medicina, per cui si pensa che sia da curare la persona che manifesta un'anomalia. Così i genitori sono convinti che si debba curare il bimbo,e purtroppo molti operatori, anche medici, agiscono in base a tale assunto implicito. Pensare alle relazioni (Sameroff, Emde, 1989), anziché a un paziente-individuo, è più complicato: per dei genitori, inoltre, è angoscioso, in quanto essi si sentono in colpa per non aver costruito una buona relazione; pensano cioè di avere sbagliato nell'illusione che la relazione avrebbe potuto essere costruita dalla volontà e dalla coscienza anziché, come invece accade, dalla loro struttura neuromentale inconsapevole. Pertanto, quando i genitori presentano il bimbo ai sanitari, credono che questi debbano curare il bambino e si sentono sconvolti quando l'operatore presenta loro una prospettiva diversa. Di qui la necessità di una formazione degli operatori che li renda competenti delle difficoltà che si manifestano a tal proposito con i genitori.

In ambito evolutivo, pertanto, ciò che si intende come psicoterapia non significa quasi mai un setting formalizzato al bimbo: psicoterapeutico è ogni intervento di sostegno o di chiarificazione che venga intrapreso nei confronti di una famiglia che si trova a fronteggiare una patologia clinica del bambino, qualunque essa sia (Missonnier, 2005).

Nell'infanzia e durante tutta l'età evolutiva sono diversi gli approcci psicoterapeutici, focali o meno, a breve o a lungo termine, individuali e più spesso familiari. Diversi approcci psicoterapeutici utilizzano un vertice teorico con un diverso modo di leggere il materiale clinico, oppure può essere diversa la modalità su cui verte prevalentemente l'azione terapeutica. In questo ambito ha fatto storia la psicoanalisi infantile. Melanie Klein per prima ha evidenziato l'importanza di una psicoanalisi precoce dimostrando come il gioco del bambino possa essere interpretato (1932). Winnicott $(1971,1977)$ pone il terapeuta come "oggetto transizionale" nella diade genitore-figlio e con i bambini più grandicelli usa gli scarabocchi, e indica come momenti "sacri" quegli istanti decisivi in cui l'intuizione dell'analista gli consente di cogliere gli aspetti più significativi, facilitatori di un cambiamento.

A partire dalla fine degli anni ' 80 i disturbi della prima infanzia, oggetto di con- 
sultazione pediatrica, vengono affrontati con modalità diverse, sia in ambito di ricerca sia di clinica. Si sta sviluppando una nuova prospettiva, orientata alla promozione della salute, che si esprime con l'esigenza di ampliare l'area della consultazione e l'organizzazione di servizi di prevenzione primaria per genitori e bambini al di sotto dei 3 anni mediante l'utilizzo di diversi operatori, in primis la psicologia clinica: con l'obiettivo di fornire un supporto valido nei momenti più critici dello sviluppo. L'intervento rivolto alle patologie della relazione può avere forme diverse: sostegno psicoeducativo, psicoterapia congiunta genitore-bambino, consultazione periodica o psicoterapia con percorsi più lunghi.

Nella consultazione della prima infanzia il focus sulla relazione mira al recupero delle competenze genitoriali e delle potenzialità di sviluppo del bambino. In ambito psicoanalitico si diffondono le terapie congiunte genitore-bambino secondo il modello proposto dalla Fraiberg, con interventi anche domiciliari (Fraiberg, 1999). La Fraiberg ha dato esempio di modalità di intervento in situazioni di rischio relazionale entrando nel contesto familiare, per supportare funzioni genitoriali carenziali o disturbate e per "liberare" la relazione con il bambino dai fantasmi del passato familiare (Fraiberg, Adelson, Shapiro, 1975). Secondo il modello della Fraiberg, nel processo del divenire genitori verrebbero risvegliati antichi conflitti irrisolti dei genitori stessi che vengono messi in atto attraverso "copioni", che ricalcano nel presente reale col bimbo i "fantasmi nella camera dei bambini" che furono vissuti dai genitori in una pericolosa riedizione transgenerazionale. Aspetti transgenerazionali che si tramandano di genitore in figlio attraverso la trasmissione di particolari stili relazionali di attaccamento e specifiche configurazioni fantasmatiche vengono, secondo la Fraiberg, attivate dalla presenza del bambino, che diviene così "oggetto di transfert" degli aspetti infantili non elaborati dai genitori.

Sono gli studi di Bowlby (1988) che ci hanno condotto alla conoscenza dell'esistenza di specifici "Modelli Operativi Interni”, implicati e deputati alla costruzione dei primi e significativi legami di attaccamento che intercorrono tra genitori e bambini. Attraverso tali modelli, di padre in figlio si veicola una trasmissione transgenerazionale delle specifiche modalità di attaccamento e, conseguentemente, del possibile disagio o della "salute mentale". Secondo Bowlby, la "microcultura familiare" che si trasmette di generazione in generazione attraverso peculiari stili di attaccamento non è meno importante della stessa eredità genetica nel determinare la salute o la patologia mentale del futuro individuo. Il divenire genitore e l'assumersi la cura del proprio figlio ha il potere di attivare funzioni mentali adeguate al nuovo compito, quali il favorire la crescita del bambino attraverso i legami di attaccamento, ma allo stesso tempo può attivare situazioni emotive che possono portare a difficoltà. Intervenire in questo ambito diventa allora estremamente significativo, proprio perché "la storia dei genitori non diventi il destino dei figli".

I lavori di Seligman (1994) di Lieberman e Pawl (1993) proseguono l'attività della Fraiberg, sottolineando il ruolo centrale della relazione terapeutica come paradigma della relazione che si vuol promuovere tra i genitori e il bambino nell'ottica del cambiamento. La Fraiberg (1980) sottolinea l'aspetto della speranza: il neonato può fornire una forte spinta verso cambiamenti positivi nei suoi genitori (rappresenta le loro speranze e i loro desideri più profondi, sostiene il rinnovamento del Sé, la sua 
nascita può essere vissuta come una rinascita psicologica per i genitori, può creare e offrire una speranza riparatrice al genitore che desidera trattare i propri bambini meglio di quanto sia stato trattato lui), ma parimenti può attivare desideri, sentimenti e impulsi "distruttivi" latenti nei genitori. Nella visione della Fraiberg appare necessario che gli aspetti progressivi dello sviluppo del bambino siano sufficientemente investiti da parte dei suoi genitori, anche se ciò purtroppo non sempre accade. Alcune persone funzionano molto bene nelle relazioni lavorative e adulte, ma trovano difficoltà negli aspetti connessi all'essere diventati madre e padre (Furman, 1966). Il diventare genitore implica un'assunzione di ruolo che comporta un intenso lavoro intrapsichico (capitolo 2).

La psicoterapia congiunta genitori-bambino viene sviluppata tenendo in considerazione modelli teorici differenti: in Francia e Svizzera (Lebovici, Soulè, Missonnier, Cramer, Palacio-Espasa) la terapia congiunta madre-bambino viene utilizzata in epoca precoce, nel periodo perinatale, ma anche durante tutta l'infanzia.

Lebovici (1983), indicando come le relazioni affettive contribuiscono alla costruzione della mente del bambino attraverso una interazione fantasmatica costituita dalla comunicazione non verbale, mette in evidenza quali sono le basi per la pratica delle consultazioni genitori-lattanti (Lebovici 1980; 1989; Lebovici et al., 1989).

Palacio-Espasa e Manzano (1982) hanno dato anch'essi contributi alla teorizzazione dell'intervento breve: l'azione terapeutica e il cambiamento si fondano su un'elaborazione fantasmatica attivata nel transfert dei genitori. L'azione terapeutica porta al cambiamento quando può essere esplicitata la relazione latente, di contenuti non consapevoli, conflitti pregressi o attuali con i propri genitori, o lutti irrisolti quali nuclei tematici più frequenti oggetto di interpretazione. Le terapie congiunte genitore-bambino proposte da Cramer e Palacio-Espasa della scuola di Ginevra (1993) mettono in evidenza come l'area delle relazioni disfunzionali può presentarsi attraverso un ripetuto "copione" di relazioni conflittuali, maltrattamenti, abusi, psicopatologie che si trasmettono da una generazione all'altra. Il cambiamento terapeutico avviene a seguito dell'interpretazione, rispetto alla "sequenza interattiva sintomatica": la conflittualità nei genitori può manifestarsi attraverso un pattern interattivo ripetitivo con il bambino. Il cambiamento terapeutico si attua attraverso il lavoro psichico che il genitore fa con l'analista e il potenziale transfert del bambino verso il terapeuta che passa attraverso il contemporaneo transfert genitoriale (PalacioEspasa, Cramer, 1989).

Le terapie congiunte genitori-bambino hanno aperto nuove prospettive di intervento e ne hanno ampliato il focus sviluppandosi prevalentemente in questi ultimi anni come consultazioni terapeutiche (Manzano, Palacio-Espasa, Zilkha, 2001). Queste consultazioni si indirizzano in modo più ampio ai vari componenti del nucleo familiare e possono essere effettuate oltre il periodo della prima infanzia attraverso interventi brevi che possono venire reiterati nel tempo. Anche i punti di vista teorici e tecnici che possono sembrare diversi nelle differenti Scuole non sono tuttavia alternativi. Stern, per esempio, mette in evidenza il transfert positivo e l'alleanza terapeutica in una particolare organizzazione psichica caratteristica del periodo perinatale: la costellazione materna. La madre ha necessità di essere sostenuta e valorizzata per riorganizzare la sua identità come madre e il terapeuta si trova a ricopri- 
re la funzione di una buona "nonna" (Stern, 1995).

La tecnica adottata dalla scuola inglese, nei trattamenti della Tavistock Clinic nell'Under Fives Counsellling Service di tradizione kleiniana e bioniana, comporta interventi più specificatamente psicoanalitici, centrati sulle competenze di ascolto del terapeuta. Nel bambino incarcerato nel sintomo si intravedono fantasmi, paure inespresse: la "pregnanza" del sintomo copre l'assenza di immaginazione. Il processo terapeutico avviene in gruppo attraverso l'attivazione di un processo di rêverie collettivo . "L'oggetto gruppale" è contemporaneamente il bimbo, i suoi genitori e i coterapeuti.

La consultazione terapeutica Tavistock si basa sul concetto bioniano di contenimento e si estende alle relazioni intrafamiliari: la relazione con il terapeuta dovrebbe fornire ai genitori un modello di funzione parentale efficace. Nella consultazione genitore-bambino questo modello implica che, nella "mente" dei genitori, l'esperienza del contenimento favorisca processi di cambiamento. La consultazione diventa un primo riferimento per dare ai genitori in difficoltà un aiuto e creare le basi per un lavoro psicoterapeutico più approfondito.

In ambito italiano altre modalità di intervento a orientamento psicoanalitico sono la "consultazione partecipata" (Vallino, 2004, 2007, 2009), intervento che si ritiene utile come preparazione a una psicoterapia. Questa modalità è un'estensione dell'Infant Observation alla clinica infantile: prevede sedute ripetute nel tempo, in cui i genitori partecipano con l'analista ai giochi del figlio per poi fare riflessioni sul significato comunicativo ed evolutivo del comportamento. Prima ancora di poter capire il bambino, c'è bisogno che il terapeuta recuperi la caduta di pensiero di genitori dominati dall'angoscia, mentre $i$ bambini si aspettano di poter essere rimessi in contatto con i loro genitori.

Modelli per il trattamento dei disturbi relazionali precoci secondo invece un vertice teorico a indirizzo comportamentale sono, ad esempio, quelli proposti da Susane McDounough (McDounough, 1992): la tecnica maggiormente utilizzata è quella della videoregistrazione che consente al terapeuta di evidenziare ai genitori quegli aspetti dell'interazione che possono attivare processi di cambiamento nel comportamento genitoriale. Questa tecnica è ritenuta una modalità non intrusiva che consente di trattare bambini con disturbi funzionali e problemi organici e genitori che manifestano resistenze. Una prima valutazione clinica del funzionamento familiare viene effettuata a domicilio e i genitori sono invitati a partecipare insieme a tutti gli altri membri della famiglia, compresa la generazione dei nonni. Questi familiari, anche se poi non entreranno nel trattamento successivo, possono comunque sostenere i genitori indirettamente. I genitori vengono coinvolti attivamente nelle osservazioni dei comportamenti durante l'interazione e partecipano alla valutazione dei progressi del trattamento e dei cambiamenti attraverso i filmati realizzati durante le sedute. I processi di cambiamento vengono commentati progressivamente durante la loro evoluzione. Questa tecnica consente un feedback di ciò che accade nella relazione: attraverso domande il terapeuta sollecita processi di riflessione e commenti sui vissuti dei genitori, sottolineando esempi specifici di comportamento positivo e di competenza genitoriale che possano consentire un cambiamento rispetto alle problematiche identificate. Un aspetto positivo della videoregistrazione è documentare i cam- 
biamenti che si manifestano progressivamente durante tutto il trattamento. Se si manifestano momenti di impasse, una visione dei filmati dei cambiamenti ottenuti nel passato potrà incoraggiare $\mathrm{i}$ genitori a proseguire nella psicoterapia. Al termine del trattamento i filmati vengono consegnati ai genitori, quale documentazione dei cambiamenti durante il corso del trattamento.

Altri approcci teorici al trattamento psicoterapeutico, come quello proposto da Brazelton (Brazelton et al., 1979), si fondano prevalentemente sulla promozione della "capacità di previsione" nel genitore (Kernberg, 1992): si focalizzano sulla qualità dell'interazione tra genitore e bambino intesa come predittiva dello sviluppo successivo del bambino e indicano che quando il genitore diventa consapevole del modo in cui stimola il proprio bambino, diventa anche più cosciente degli effetti prodotti dalle sue azioni sullo sviluppo stesso del bambino. Il comportamento interattivo del genitore riflette la sua adeguatezza a sostenere lo stadio evolutivo attuale del bambino, ma implica anche la possibilità di anticipare imminenti cambiamenti evolutivi. Viene definito "comportamento di previsione" la capacità genitoriale di promuovere e sostenere lo sviluppo del bambino, attraverso capacità intuitive (Papousek, Papousek, 1975) di sintonizzazione affettiva (Stern, 1985) e processi di empatia (Hoffmann, 1994), in modo da capire i segnali e i comportamenti del bambino e di prevederne l'evoluzione favorendone l'organizzazione. Il comportamento di previsione implica una capacità del genitore di attribuire un significato alla comunicazione e al comportamento del bambino e di rispondergli trasformando i segnali in messaggi significativi e riconoscibili per favorire una risposta adeguata nel bambino. Questi interventi terapeutici hanno come obiettivo la promozione di un'interazione primaria di comportamenti adeguati in modo che questi costituiscano una struttura di sostegno, uno scaffolding allo sviluppo del bambino.

\subsection{La consultazione terapeutica prenatale: I'anticipazione preventiva come fattore protettivo}

L'area di possibile attivazione di cambiamento prima della nascita è quella relativa alla perinatalità psichica dei genitori, in primo luogo costruendo percorsi di prevenzione, protettivi di eventuali patologie che potrebbero evolvere in modo anche grave.

L'intervento dello psicologo clinico perinatale, con una coppia o con il singolo genitore, può avvenire non solo su richiesta dei genitori interessati, ma anche attraverso una segnalazione di opportunità da parte di altre figure professionali (ginecologo, pediatra, ostetrica, puericultrice). Il suo compito, in questo caso più difficile, potrà allora svolgersi attraverso una supervisione con gli operatori che si occupano della nascita, in uno spazio condiviso e in sintonia.

Come abbiamo cercato di evidenziare nei precedenti capitoli (capitoli 2 e 3), il processo mentale della genitorialità è una "costruzione" che si sviluppa evolutivamente nel corso dello sviluppo dell'individuo (Bydlowski, 1989) attraverso l'infanzia, l'adolescenza e l'età adulta: per quanto riguarda la madre, la presenza di conflittualità o di antiche ferite può riflettersi nel livello di tolleranza materna ai cambiamen- 
ti psicofisiologici della gestazione, nelle sue interazioni con il feto, nelle anticipazioni del possibile dolore del parto e sarà rispecchiata da eventuali complicanze ostetriche e pediatriche (Bydlowski, Rauolu Duval, 1978). La fragilità della donna durante la gestazione è una potenziale fonte di pericolo, ma contemporaneamente anche di creatività se adeguatamente supportata da un sostegno psicologico, che potrà consentire ristrutturazioni psichiche benefiche (Cramer, Palacio-Espasa, 1993).

La nascita di un bambino veicola un triplice significato: nascita in senso biologico, nascita come psiche del bambino e nascita della rappresentazione della psiche del bambino nella psiche dei genitori (Carel, 1990). La genitorialità implica la costituzione di un involucro psichico con una triplice capacità di contenimento: della propria storia individuale, di quella coniugale e di quella transgenerazionale. Nel bambino un Sé "psicofisiologico" prenatale (Mancia, 1989) potrà svilupparsi entro una continuità biologica verso un "Sé emergente" (Stern, 1989), di neonato, se la nascita avviene entro un "ambiente" sufficientemente buono e contenitivo (Winnicott, 1965). Vi sarebbe una continuità nella linea dello sviluppo dalla prenatalità alla perinatalità facendo riferimento "all'Individuo" del periodo antecedente la nascita (Winnicott, 1966) con la possibilità di una continuità tra la funzione di contenimento interno durante la gestazione, nell'utero materno, a quella di un contenimento dell'ambiente esterno (Golse, 1995). A questi concetti Missonnier aggiunge quello di funzionamento psichico "materno prenatale placentare", per evidenziare la capacità funzionale di una gestazione psichica di contenimento e di interazione da parte della madre nei confronti del prodotto del concepimento (Missonnier, 2005).

Nella consultazione terapeutica postnatale "il bimbo è presente", con i genitori e con le loro proto rappresentazioni: nella consultazione terapeutica prenatale il "bimbo è virtuale" (Missonnier, 2005), ma può ugualmente essere investito di una relazione carica di identificazioni proiettive, in cui si sviluppa l'attaccamento. La consultazione terapeutica prenatale può consentire uno spazio e un tempo in cui sia possibile per i genitori rivedere i problemi interiori riattivati durante la gestazione, e così essere poi in grado di ripristinare una realtà con il loro bambino liberandolo dalle identificazioni distorte derivate dalla propria storia generazionale. Come in uno scenario teatrale, il setting della consultazione può consentire ai genitori di esprimere le proprie identificazioni proiettive, mentre il terapeuta come un regista ne assicura il contenimento.

Missonnier fa riferimento a quattro postulati teorici che il terapeuta deve tenere in considerazione nel corso della consultazione prenatale:

1. il bambino occupa un posto intermedio tra lo spazio intrapsichico e quello extrapsichico genitoriale;

2. il feto non va considerato come estensione narcisistica materna fino al termine della gravidanza, perché secondo l'autore il feto è un "bambino virtuale" con cui la madre stabilisce una relazione di narcisismo semmai si può parlare in casi particolari;

3. gli aspetti transgenerazionali della dinamica familiare devono essere considerati valutando la stratificazione di rappresentazioni transgenerazionali presenti in ogni filiazione;

4. la maggiore trasparenza psichica favorita dai processi della gestazione può riattivare conflitti antichi con i propri genitori. 
È pertanto indispensabile supportare quei genitori che hanno avuto esperienze traumatiche durante il periodo della gestazione.

La durata temporale della consulenza terapeutica prenatale può variare da un intervento specifico breve di alcuni colloqui in seguito a una crisi genitoriale a una terapia breve, da 3 a 10 sedute, fino a una più lunga terapia psicoanalitica. Il terapeuta opera prevalentemente attivando un contesto di contenimento, attraverso una presenza empatica di condivisione degli affetti. L'intersoggettività verbale e non verbale e l'empatia sono i principali strumenti. Il setting delle consultazioni terapeutiche prenatali è spesso quello istituzionale dei reparti di maternità ospedalieri: pertanto, il contenimento terapeutico fa parte di un "meta-contenimento" istituzionale più vasto.

Il lavoro del terapeuta è orientato a mantenere una continuità che consenta ai genitori, quando verrà il momento della nascita, di poter proseguire nell'elaborazione di vissuti che sono stati già loro "anticipati" e che potranno ora essere messi a confronto con la realtà. Missonnier fa così riferimento a strategie di "anticipazione preventiva" che vengono mobilitate dall'intervento terapeutico: i genitori vengono aiutati a metterle in scena entro un contenitore protettivo. Lo sviluppo di questa funzione di "anticipazione" della genitorialità, a partire dal periodo prenatale, è fondamentale perché consente di attivare preventivamente il cambiamento che avverrà nella transizione dalla genitorialità prenatale alla genitorialità postnatale, aiutando i genitori a fronte di eventuali sopravvenenti problematiche psicosomatiche, ostetriche, pediatriche. Le dinamiche del presente della gestazione e delle esperienze del passato, prossimo o remoto dell'infanzia dei genitori, sono presenti anche durante il parto e nel dolore a esso collegato. La percezione del dolore è notevolmente condizionata dai processi psichici della donna, attivati nel momento e nelle condizioni del parto stesso (Imbasciati, Dabrassi, Cena, 2011, cap. 6). Il supporto di un sostegno perinatale può consentire un cambiamento: il primo richiesto deriva dal confronto tra il bambino immaginario (Lebovici, 1992) e il bambino reale, attraverso una rappresentazione " anticipatrice" del proprio bambino (Perard-Cupa et al., 1992). La ricongiunzione potrà essere semplice se il bambino non presenta anomalie: l'instaurarsi invece di patologie, prenatali e perinatali, o una diagnosi prenatale infausta, rendono questo percorso molto complesso e il cambiamento difficile e doloroso.

Il percorso che conduce alla genitorialità può prendere direzioni diverse e imprevedibili. Se si presenta un handicap o un problema psicofisico alla nascita del bambino, un fattore preventivo di eventuali disturbi psichici che possono insorgere nei genitori a seguito di un infelice evento risiede proprio nella funzione protettrice di una "anticipazione", intesa come capacità di immaginare eventuali fatti imprevisti. Questa capacità può essere già presente nella mente del genitore o può venire sollecitata e supportata da interventi di consulenza o psicoterapeutici.

L'anticipazione preventiva è considerata una funzione importante che ritroviamo comune e trasversale a diversi vertici teorici di riferimento (Brazelton et al., 1979; McHale, 2010).

La "conoscenza anticipatrice", così come viene definita da Stern (Stern, 1977), è quella capacità mentale di affrontare l'imprevisto, che può presentarsi con sorprese nefaste senza per questo che il soggetto ne debba annichilito e può essere accostata alla "capacità negativa"descritta da Bion (1970) di saper stare nell'incertezza, nel dub- 
bio. Questa capacità implica riuscire a tollerare la decostruzione del già saputo, per trovarsi di fronte al "non sapere", fino a che lentamente emergano nuove possibilità di senso, fino ad allora insospettabili. Di fronte a un evento critico come una diagnosi prenatale infausta ( a seguito di una malformazione, di un'anomalia genetica, oppure di una nascita pretermine) la capacità genitoriale di anticipazione è un indicatore preventivo nei confronti di eventuali disturbi psichici dei genitori, ad esempio la depressione podst-partum, ma rappresenta soprattutto un fattore protettivo per il bambino. La capacità di anticipare implica saper attivare un cambiamento, immaginare un futuro eventualmente diverso da quello progettato e prevedere le proprie reazioni emotive e comportamentali in relazione alle conseguenze che potrebbero eventualmente accadere: è fare ipotesi sulle diverse soluzioni possibili e sulle proprie risposte (Ionescu et al., 1997).

Il concetto di anticipazione è sottinteso anche alla teoria dell'attaccamento di Bowlby (1988) con i modelli operativi interni: il bimbo attraverso i MOI ha dei modelli di relazione di sé con l'altro che gli consentono di anticipare le modalità relazionali con altri individui. Analogamente i MOI agiscono nei genitori.

Così pure il concetto di anticipazione preventiva è stato operazionalizzato attraverso una procedura sperimentale prenatale (Fivaz et al., 2002) negli interventi psicoterapeutici sistemici (Fivaz et al., 1999). Questa procedura consente di indagare le modalità relazionali che i genitori prevedono di adottare con il futuro bambino.

Nello studio della transizione alla genitorialità, Fivaz-Depeursinge ha rivolto l'attenzione alle interazioni triadiche madre-padre-bambino (Fivaz-Depeursinge, Corboz-Warnery, 1999). Dal punto di vista teorico, il riferimento è a un approccio intersoggettivo sistemico-familiare secondo cui la triade viene definita come un sistema co-evolutivo non riducibile alla somma dei sistemi diadici che la compongono. In questa prospettiva vengono studiate le interazioni triadiche già a partire dalla gravidanza, che rappresenta il momento di preparazione all'emergere della triade relazionale (Fivaz-Depeursinge, Corboz-Warnery, 1992). Le rappresentazioni che si animano nella mente dei genitori tra di loro durante la gravidanza e nella loro relazione con il proprio bambino futuro organizzano le interrelazioni che essi avranno poi con il neonato (Stoleru, 1989; Stoleru et al., 1985; Ammaniti, Stern, 1991).

A tal scopo Fivaz-Depeursinge ha sviluppato una procedura osservativa che consente di valutare la qualità delle interazioni del sistema triadico padre-madre-bambino già in gravidanza, prima che compaia il bambino reale della triade. Tale procedura è denominata il Lausanne Triadic Play (LTP) (Fivaz-Depeursinge, Corboz -Warnery, 1999) e prevede una situazione di interazioni con l'utilizzo di una bambola al posto del bambino, con un successivo confronto al quarto mese post partum una volta nato il figlio. Ciò consente di predire i comportamenti che poi i genitori avranno con il loro neonato e di verificarlo dopo la nascita. L'interazione dei genitori tra loro e con il bambino durante la gestazione viene così osservata in laboratorio e poi in diversi altri momenti dopo la nascita. La coppia genitore-bambino e la triade madrebambino-padre vengono considerate dei sistemi tra loro interdipendenti. Le osservazioni vengono videoregistrate e analizzate con modalità di microanalisi. L'intervento ha spesso luogo all'interno di una rete terapeutica, secondo l'approccio sistemico, in cui spesso vengono coinvolti diversi professionisti (pediatra, infermiere, psi- 
cologo, neuropsichiatra). La terapia è multifocale, cioè può orientarsi su molteplici aspetti dell'interazione, dal conflitto all'accordo: il terapeuta entra nel sistema con la modalità ritenuta più pertinente, sia in fase di osservazione e proposta preventiva che in fase di attuazione della situazione terapeutica. L'approccio della Fivaz ha come obiettivo quello di attivare le risorse della famiglia anticipandone le rappresentazioni prima che il bimbo agisca, esercitando in tal modo una funzione preventiva nel promuovere una co-evoluzione (Fivaz et al., 1992) del sistema genitoriale e del sistema bambino.

\subsection{Gli organizzatori psichici del cambiamento perinatale genitoriale: funzione simbolica degli esami medici e delle consultazioni ostetrico/ginecologiche}

Secondo Missonnier (2005) i controlli medici nel periodo perinatale sono anche "organizzatori psichici” che declinano il cambiamento nel processo di genitorialità. I momenti di consultazione e di confronto con medici, ostetriche e altri operatori della nascita, attraverso le indagini di routine specialistiche che vengono effettuate durante la gravidanza, parto e post partum, sollecitano processi di simbolizzazione che scandiscono il percorso interiore della gestazione, nascita, parto e puerperio, accompagnando l'evoluzione psichica della genitorialità.

I rituali connessi alla nascita esistono da sempre, come una funzione simbolica protettiva nei confronti della donna del nascituro e della famiglia. Questo ruolo nella nostra attuale società viene svolto ed efficacemente rinforzato da tutta la serie di controlli sanitari cui ben volentieri le donne e le coppie si sottopongono: essi svolgono, oltre le loro funzioni sanitarie, anche un ruolo rituale che come tale, inquadrato nel sociale, ha funzioni di "organizzatore psichico" nel processo di genitorialità (Missonnier, 2005). I reparti maternità sono uno spazio rituale di iniziazione, in cui più figure professionali intervengono ed esercitano cooperativamente una funzione preventiva delle angosce attraverso una elaborazione creativa e condivisa della nascita.

Nel periodo prenatale tra gli organizzatori psichici l'ecografia rappresenta un punto di incontro tra professionisti-feto-genitori e operatori, un luogo privilegiato per una "riflessione" pre-natale (Missonnier, 2005): ciò induce nei genitori un confronto con il feto reale e una risonanza con il bambino immaginato, retaggio della storia individuale, coniugale e generazionale. Le valenze emotive dell'immagine ecografica si collocano tra due polarità: confermano il processo di genitorialtà e le funzioni di contenimento, oppure conducono a una crisi psichica, grave di identificazioni proiettive patologiche, nei casi di anomalie. In questi ultimi anni l'ecografia consente l'accesso a una consultazione psicoterapeutica che può consentire la metabolizzazione genitoriale delle inquietanti ripercussioni inerenti la rivelazione e fornire un supporto dinamico dell'anticipazione del bambino. Su questi vissuti si potrà elaborare un percorso psicoterapeutico.

L'ecografia può diventare anche uno spazio transizionale, luogo di transizione (Pe- 
ruchon, 1997), per l'elaborazione di vissuti relativi alla salute del bambino. Nella donna si attua l"'anticipazione immaginaria" che la conforta come un "organizzatore psichico" contro le angosce spesso inevitabili di eventuali malformazioni del nascituro, le quali di per se stesse possono avere potenzialità strutturanti o distruttive, a seconda dei conflitti della genitorialità (Cramer, Palacio-Espasa, 1993). Alcuni autori fanno riferimento ai significati positivi che possono assumere anche le angosce rispetto al feto, in quanto rappresentano un aspetto del legame di attaccamento materno: la loro mancanza potrebbe significare al contrario un segno negativo di tale attaccamento (Leifer, 1977). In assenza di anomalie fetali questi timori sono interpretati sia come una manifestazione di difficoltà del processo della maternità, sia come una "anticipazione creatrice" che tutela dalla imprevedibilità di eventuali fatti traumatici.

Anche una diagnosi prenatale viene considerata come altro "organizzatore psichico" a funzione protettiva e permette di condurre a un accompagnamento perinatale multidisciplinare integrato e a un servizio di consultazione e di sostegno terapeutico condiviso.

Il confronto con il neonato reale è considerato un "organizzatore psichico". La depressione post partum funziona anch'essa come organizzatore psichico, nei casi meno favorevoli, in cui non si sia riusciti a evitarla: essa rappresenta un momento di ritiro psichico dalla realtà che reclama aiuto, che in tal modo può essere offerto alla donna.

Il dolore del parto può fungere come "organizzatore psichico": comporta un dolore fisico e un dolore psichico. Il timore del dolore comporta la richiesta di analgesia: Missonnier (2005) evidenzia come un parto programmato per convenienza personale e non per obbiettivi medici sottintende una vulnerabilità psichica che può costituire fattore di rischio, e che come tale va presa in considerazione. L'autore indica pertanto come sia necessario comprenderne il significato e favorirne una sua eventuale elaborazione.

Le richieste di parto programmato per convenienza personale sottintendono una domanda di aiuto, mascherata, e costituiscono un indicatore prenatale di un disagio che ha necessità di essere elaborato. Il controllo difensivo sotteso non viene spesso percepito, perché avviene una collusione con analoghi vissuti negli operatori della nascita.

L'evento parto ha il significato organizzatore di una separazione tra la donna e il bimbo fino ad allora considerato quasi parte di sé.

La nascita prematura o con patologie del neonato, sono anch'essi "organizzatori psichici" che comportano per il genitore una doppia separazione: quella della nascita e quella dell'allontanamento del neonato in un reparto di patologia intensiva neonatale. Il ricovero del neonato in una UTIN spesso impedisce una simbolizzazione e un'elaborazione della nascita dei genitori come tali. La loro sofferenza può essere molto elevata anche se nascosta. Di fronte al trauma della doppia separazione, quella della nascita a seguito del parto e quella del ricovero del bambino nato pretermine, i genitori non hanno la possibilità psichica di elaborare il lutto per la perdita, come avviene in una nascita a termine in cui si attiva invece un processo di ricomposizione del bambino immaginato nel bambino reale. Il bambino ricoverato è un bambino distante, difficilmente raggiungibile anche a livello sensoriale e questo rende molto più complesso il processo di simbolizzazione della separazione. 


\subsection{Integrazione tra ricerca e clinica per monitorare il cambiamento}

Considerazione particolare va dedicata alla teorizzazione e alla modalità di intervento operata da Stern nel modello teorico della Costellazione materna (1995). Nel periodo perinatale la madre e il padre si trovano in una situazione psichica particolare, un'organizzazione unica della vita psichica genitoriale, che fa del genitore un "paziente" speciale. L'alleanza terapeutica entro cui può trovarsi a lavorare un terapeuta è pertanto diversa. Con la gravidanza e la nascita del figlio cambiano gli interessi, le paure e le preoccupazioni: i pensieri materni sono focalizzati su uno stato affettivo particolare, di innamoramento del bambino e in questo le madri hanno necessità di essere sostenute. La Costellazione materna è un costrutto costituito da quattro tematiche in cui si organizzano le relazioni interpersonali della madre secondo i suoi desideri, paure, ricordi e sentimenti. Questo costrutto può essere sintetizzato nel tema "vita, crescita-sviluppo del bambino", in quello della "relazionalità primaria genitore-bambino", della "matrice di supporto sociale alla genitorialità" e in quello della "riorganizzazione dell'identità del genitore". La Costellazione materna comprende anche una "trilogia" di preoccupazioni specifiche del legame della madre con sua madre, il rapporto della donna con se stessa in quanto madre e il rapporto con il suo bambino. In ognuna di queste aree, che la ricerca ha posto in luce, occorre indagare nella clinica del singolo caso se si vuole porgere aiuto alla donna che ne abbia bisogno.

La relazione della madre con il suo bambino è l'organizzazione psichica più pregnante della Costellazione materna: il transfert che si sviluppa in questa situazione consiste nell'elaborazione del desiderio della donna di essere supportata e aiutata da un'altra figura con funzioni materne. Anche in contesti non terapeutici le madri cercano altre donne, come l'ostetrica, l'infermiera, la puericultrice che condividano la loro esperienza personale fornendo sostegno e incoraggiamento (Winnicott, 1987). Nella situazione terapeutica questa ricerca di aiuto viene rappresentata dalla figura del terapeuta attraverso il transfert che Stern (1985) denomina "transfert della buona nonna": il terapeuta interviene con consigli, visite a casa, orientando la terapia più sugli aspetti positivi che non sulla patologia.

L'approccio terapeutico secondo Stern può essere descritto in base a tre aspetti:

- la scelta del punto di ingresso nel sistema relazionale valutato come pregnante (per esempio le rappresentazioni dei genitori, il comportamento del bambino, l'interazione madre-bambino, il comportamento della madre);

- la scelta del bersaglio che il terapeuta si propone di modificare (per esempio il comportamento della madre oppure le rappresentazioni dei genitori);

- le modalità di trattamento (interpretazione, chiarificazione, rinforzo, educazione, sostegno, consiglio).

Le terapie madre-bambino sono in genere brevi e contano dalle 3 alle 12 sedute, di solito settimanali. Qualora si rendesse necessario un tempo più lungo, si suggerisce una psicoterapia individuale per la madre. Dato che il sistema relazionale che è stato oggetto di intervento può ripresentarsi in forma diversa in diverse fasi dello sviluppo, la terapia può essere ripetuta una o più volte a distanza di tempo. 
Stern fa riferimento a due concetti propedeutici alle consultazioni terapeutiche: il "bambino osservato" e il "bambino clinico" (Stern, 1985). Questi due concetti sono da considerarsi trasversali e complementari a tutti gli interventi terapeutici . Con "bambino osservato" l'autore indica l'importanza della ricerca e dei dati conoscitivi derivati dagli studi della psicologia dello sviluppo, attraverso setting di osservazione sperimentale che vanno conosciuti anche dal terapeuta clinico. Con "bambino clinico" richiama le rilevazioni della clinica e i modelli psicopatologici dell'infanzia, con riferimento alla "indagine" e alla "ricostruzione" che il terapeuta deve saper utilizzare per attivare un' adeguata proposta terapeutica. Attraverso questi due concetti Stern propone un'integrazione tra ricerca e clinica per monitorare il cambiamento nell'evoluzione del trattamento: gli sviluppi della ricerca possono dare un contributo per la comprensione degli aspetti clinici. L'autore propone pertanto un modello terapeutico che opera in modo complementare e integrato (Stern, Bruschweiler, 1989), facendo riferimento al modello conoscitivo (bambino osservato) e a quello di intervento (bambino clinico) in cui sono utilizzate in modo circolare l'osservazione dell'interazione reale e l'indagine clinica retrospettiva. In ogni intervento clinico occorre tenere in considerazione l'ottica delle due prospettive, osservazione reale e clinica, contemporaneamente presenti in ogni interrelazione dinamica. Il bambino osservato consente una comprensione dello sviluppo infantile e delle relazioni attuali; il bambino clinico consente di accedere alla complessità e alla ricchezza del mondo interno intrapsichico e relazionale infantile. Sono due vie di accesso complementari ed entrambe necessarie per i trattamenti in ambito perinatale: l'osservazione del bambino consente di registrare cambiamenti osservabili percettivi, motori, conoscitivi, emotivi, reattivi, utilizzando metodologie sperimentali di videosservazione; attraverso il concetto di "bambino clinico" si intendono le complessità di indagine e di comprensione delle motivazioni del comportamento, delle dinamiche intrapsichiche e interpersonali, delle rappresentazioni e dei significati emotivi dell'esperienza. In tal modo Stern descrive gli interventi precoci in ambito perinatale come nuove modalità di trattamento per una "nuova popolazione clinica" (Stern, 2006), che ha esigenze diverse rapportate al periodo dello sviluppo che sta attraversando il bimbo. Tale modalità va esaminata nella sua realtà e nel suo contesto clinico al di là di valutazioni consuete per altri contesti terapeutici.

\subsection{I fattori fondamentali di cambiamento comuni alle diverse vie di accesso all'intervento}

Stern mette in evidenza come nella maggior parte degli approcci terapeutici precoci i risultati siano positivi e rileva come in tutte le terapie ci siano fattori comuni che consentono di raggiungere risultati efficaci: si tratta di "fattori non specifici", non sempre facili da individuare nella singola terapia. Questi fattori vengono definiti più propriamente "fattori fondamentali", ritenuti i più importanti, e ne vengono sottolineati quattro: 
1. il tipo di relazione clinica in cui interviene il terapeuta;

2. le caratteristiche del transfert;

3. l'elaborazione del processo terapeutico nel contesto di sviluppo e

4. gli aspetti clinici positivi.

Il primo fattore riguarda la relazione genitori-bambino-terapeuta. Il terapeuta può intervenire con il singolo individuo come "paziente" oppure considerare "paziente" la relazione e intervenire sul mondo rappresentazionale e sulla sua influenza nell'interazione. Il secondo fattore comune ai diversi approcci terapeutici è costituito dal transfert, che viene tenuto in considerazione con modalità diverse a seconda dei vertici teorici di riferimento: in ambito non psicoanalitico si parla di alleanza terapeutica in relazione a un transfert positivo. Analogamente il controtransfert, strumento fondamentale per il terapeuta, consente al clinico di avvicinarsi alla propria risonanza rispetto ai disturbi della relazione.

Il terzo fattore segnalato da Stern è caratterizzato dal cambiamento dovuto al processo terapeutico nel contesto del bambino, attraverso processi di elaborazione che si sviluppano con modalità "verticali", cioè progressivamente nel tempo: il parametro di riferimento per il terapeuta è un trattamento breve che può essere reiterato nel tempo, longitudinalmente, durante le diverse fasi dello sviluppo e della crescita del bambino. La maggioranza delle psicoterapie, durante il periodo perinatale, hanno una durata breve: questa modalità molto spesso comporta che l'intervento debba venire ripetuto a cicli periodici, magari dopo un intervallo di alcuni mesi. Nel trattamento con gli adulti è necessaria una "elaborazione" del materiale clinico, perché gli effetti siano più duraturi. In ambito evolutivo invece, e ancor più in ambito perinatale, quanto più il bambino è piccolo tanto più è impossibile poter attivare un processo di elaborazione prolungato nel tempo, a meno che si tratti di un intervento di psicoterapia individuale. Risulta più funzionale all'accompagnamento dei genitori, nel loro processo di evoluzione della genitorialità e di crescita del bambino, rivederli a periodi ciclici, quando devono affrontare nuove tappe dello sviluppo del bambino e possono incontrare difficoltà non ancora esplorate.

Così in ambito perinatale si verifica la possibilità che i genitori si presentino nei primi mesi di vita del bimbo per una consulenza in relazione a una problematica, ad esempio del sonno, e il cambiamento possa verificarsi abbastanza velocemente; dopodiché la famiglia può ritornare dopo il primo anno per un altro problema relazionale. Anche in questo caso potrebbe essere possibile ottenere un cambiamento con alcune settimane di trattamento. I genitori possono ritornare ancora successivamente con altre problematiche, a seconda del periodo di sviluppo che sta attraversando il bambino: il problema può essere sempre lo stesso, ma ripresentarsi con sfaccettature diverse. Se non è possibile subito una risoluzione, spesso è necessario aspettare che si abbia accesso alle diverse aree di sviluppo prima di poter accedere a un'elaborazione che possa essere assimilata, compresa e possa esitare nel cambiamento.

Le psicoterapie in ambito perinatale, intese come "consultazione terapeutica perinatale" (Missonnier, 2005), fanno riferimento più propriamente a consultazioni, più che a procedimenti terapeutici. Ciò sottolinea il fatto che può rendersi necessario reiterare l'intervento secondo le modalità insite ai processi di sviluppo che 
caratterizzano il periodo evolutivo in cui viene richiesto.

Brazelton (Brazelton, Als, 1979), indica che nella transizione a ogni nuova fase di sviluppo il bambino e i suoi genitori sono orientati al cambiamento: per questo le psicoterapie in ambito perinatale sono interventi e/o consultazioni terapeutiche a breve termine. Il termine breve può essere riferito al numero ridotto delle sedute terapeutiche (3-10) oppure alla durata nel tempo. Gli interventi ripetuti nel tempo consentono ai bambini e ai loro genitori di accedere a elaborazioni progressive. Talora ciò che in altri termini potrebbe apparire come un cambiamento terapeutico fallito assume invece connotazioni ottimali in un contesto di sviluppo. Quello che in ambito perinatale e della prima infanzia può essere ottenuto con trattamenti ripetuti, nel tempo ha la stessa efficacia di quei trattamenti continui a lungo termine in altre situazioni cliniche. In ambito di consultazione perinatale e di trattamenti della prima infanzia, la caratteristica di un'elaborazione ottenuta verticalmente fa sì che alla fine di ogni fase del trattamento il transfert non venga risolto o ridotto, così che i genitori possano gestire i periodi di interruzione tra le fasi del trattamento. Queste modalità, caratteristiche dei trattamenti nella prima infanzia, non vanno considerate con connotazioni negative o come incomplete, ma come una terapia in cui il trattamento e i cambiamenti avvengono a tappe successive, intese come una necessità insita nella speciale popolazione clinica, che è in fase di sviluppo.

Una modalità per aiutare $\mathrm{i}$ genitori nei periodi tra le fasi di trattamento è la non risoluzione del transfert e/o il mantenimento di un transfert positivo. Con queste modalità è possibile al terapeuta accompagnare la famiglia che in genere ritorna per riprendere il trattamento, non con vissuti di fallimento per il precedente intervento, ma per trovare nuove modalità di risoluzione ad aspetti problematici presentati dalle nuove fasi di sviluppo del bimbo.

Il quarto fattore comune alle psicoterapie nella prima infanzia è di porre l'enfasi su ciò che è "sano" rispetto ciò è che patologico nel quadro clinico, in relazione al processo di sviluppo. Il sistema genitore-bambino è proteso al cambiamento dal processo di sviluppo stesso, insito al sistema in evoluzione. Negli adulti la forza dello sviluppo è inferiore, i desideri di benessere, lo stato di disagio e di sofferenza sono i principali motori del cambiamento: nel sistema genitore-bambino questi elementi sono secondari alle spinte di base della crescita, intrinseca allo sviluppo a cui propende l'individuo.

Un'ulteriore motivazione a considerare più gli aspetti positivi che quelli negativi è che, per la rapida progressione dello sviluppo, l'intero sistema genitore-bambino è sottoposto a riorganizzazione in diversi momenti di transizione delle fasi dello sviluppo. Proprio queste riorganizzazioni, che fanno parte dei processi di sviluppo, possono favorire una maggiore apertura al cambiamento insita nel sistema stesso. Il maggior sostenitore di questo concetto è Brazelton (1992): 1'autore indica come il sistema di base sia di per sé più aperto al cambiamento, anche per il fatto che periodicamente e in prossimità di fasi che si susseguono e che sono caratteristiche dei processi di sviluppo si presentano nuovamente periodi di maggiore apertura. La modalità relativa ai trattamenti brevi e ripetuti è pertanto considerata la più adatta alle situazioni di sviluppo nella prima infanzia. 


\subsection{Modello Dinamico di Interdipendenza di Stern: un'integrazione tra i diversi interventi perinatali}

Un ulteriore concetto proposto da Stern è il Modello Dinamico di Interdipendenza. Negli approcci terapeutici precoci può essere molto complesso identificare chi sia veramente il paziente su cui prevalentemente indirizzarsi al fine di modificare alcune interazioni: quando sono presenti disturbi precoci della relazione, spesso il ruolo del bambino come portatore di disturbo è per i genitori il fulcro che per essi dovrebbe direzionare tutto il trattamento e che, secondo le loro aspettative, li conduce dallo specialista. Tuttavia, secondo Stern, non è così fondamentale che sia notificato chi è il paziente o a chi sia indirizzata principalmente la terapia perché essa si sviluppa comunque se il terapeuta è esperto, all'interno del contesto evidenziato dal modello genitore- bambino. È questo il primo fattore comune a tutti gli approcci terapeutici: i soggetti costituiti dai singoli, la madre, il bambino e il padre sono considerati tutti elementi di un sistema interdipendente e in costante interazione dinamica.

Il cambiamento può implicare modificazioni anche in uno solo degli elementi del sistema, ma tutti gli altri elementi ne vengono coinvolti, per adeguarsi alla modificazione intervenuta nel primo. Non ha molta importanza il punto prescelto per entrare nel sistema con la terapia: una volta innescato un cambiamento, tutto il sistema sarà comunque coinvolto nelle modificazioni. Possono essere possibili molte vie di accesso al cambiamento, determinate dal vertice teorico di riferimento del terapeuta (psicoanalitico, sistemico, comportamentista, teoria dell'attaccamento), ma il cambiamento all'interno del sistema è determinato dalla natura del sistema stesso (Stern, 1992).

Questo implica che approcci anche molto diversi possono avere uguali opportunità di promuovere efficacemente, e con successo, il cambiamento.

L'esperienza soggettiva della terapia e delle sue modalità di cambiamento sono comunque differenti: Stern riporta l'esempio del confronto tra due modalità terapeutiche con punti di entrata molto diversi, in cui i risultati terapeutici si sono manifestati con modalità abbastanza simili. L'approccio psicoanalitico di Ginevra (PalacioEspasa, Manzano, 1982; Manzano et al., 2001) è rivolto prevalentemente a intervenire nelle rappresentazioni dei genitori, mentre l'approccio della McDonought (1992, 2006) a orientamento comportamentista educativo è rivolto prevalentemente al cambiamento del comportamento genitoriale nell'interazione. Le aspettative relative al primo approccio sono riferite a un cambiamento delle rappresentazioni genitoriali, mentre per l'altro sono prevalentemente rivolte al comportamento nella relazione. Entrambi i trattamenti portano però a cambiamenti sia nelle rappresentazioni genitoriali che nei comportamenti, evidenti dalle interazioni che vengono videoregistrate (Cramer, 1990). La ragione di questi risultati sta secondo Stern nella presenza di un "modello dinamico di interdipendenza", che trasferisce all'interno dell'intero sistema genitore-bambino l'intervento terapeutico applicato in un solo punto.

Il modello a orientamento psicoanalitico, prevalentemente focalizzato sulle rappresentazioni genitoriali, prende in considerazione le proiezioni, le identificazioni, le attribuzioni, le paure, i desideri, i conflitti, le fantasie del mondo rappresentazio- 
nale genitoriale, "attivo" nell'interazione col bambino (Stern, 1991). Non tutto il mondo rappresentazionale dell'adulto viene considerato, ma solo gli aspetti più significativi per i problemi clinici della relazione col bambino. Secondo Stern nell'interazione del comportamento genitoriale sono presenti queste "rappresentazioni attive": è possibile una loro identificazione attraverso l'osservazione clinica (Stern, 1971; Cramer, Stern, 1988; Cramer, 1990). I comportamenti intervengono per mantenere o rinforzare le rappresentazioni attive genitoriali. Il cambiamento del mondo rappresentativo genitoriale diventa evidente perché rilevabile attraverso i comportamenti sensibili che questi rivolge al bambino.

I diversi vertici di riferimento teorico e le rispettive modalità terapeutiche sono comunque orientate al cambiamento, che può essere rivolto prevalentemente a un elemento individuato come "bersaglio" (Stern, 1992): anche se le teorie sono diverse agiscono comunque sullo stesso "sistema funzionale" genitore-bambino. Ciò che diversifica gli approcci terapeutici sono i diversi punti di entrata nel sistema.

\subsection{Le diverse "vie d'ingresso" nella consultazione terapeutica secondo la Teoria dell'Attaccamento}

Nell'ambito del paradigma teorico dell'attaccamento recenti studi (Oppenheim, Goldsmith, 2010) fanno riferimento a diverse modalità di ingresso alla terapia, intese come opportunità di intervento in cui possono venire utilizzate strategie e modalità differenti, rivolte alle difficoltà relazionali genitore-bambino, che possano attivare esiti positivi nel trattamento. Oppenheim e Goldsmith (2010) individuano una strategia definita come "risoluzione" (Oppenheim et al., 2010): processo di rielaborazione a livello rappresentazionale e comportamentale in cui avviene una ri-organizzazione di quei modelli operativi interni che risultano inadeguati alle situazioni relazionali attuali con il bambino, in seguito a un cambiamento delle condizioni esterne, come lutti o traumi.

Le diverse modalità di intervento operate secondo i vertici di riferimento della teoria dell'attaccamento che hanno come obiettivo comune la "risoluzione", possono agire secondo due livelli: attraverso quello del sistema rappresentazionale genitoriale (Fraiberg, 1980) e quello del sistema comportamentale dei genitori. Sono due vie di accesso differenti, ma che possono condurre a esiti terapeutici simili perché gli aspetti delle rappresentazioni e del comportamento sono interconnessi e i cambiamenti che intervengono a livello di un sistema hanno ricadute sull'altro (Stern, 1995; Sameroff et al., 2004); non è pertanto determinante la via di accesso scelta per l'intervento, se non solo in quei particolari casi in cui determinati fattori di cambiamento positivi risultino invece vincolati a una specifica via di accesso piuttosto che ad un'altra.

Il focus dell'intervento viene comunque rivolto al genitore, considerando che l'adulto si possa rendere maggiormente responsabile, nell'impegno della interazione, a prendere in considerazione aspetti del proprio comportamento nell'ottica di rivedere quelli problematici e inadeguati nei confronti del bambino. Il cambiamento è 
prevalentemente vincolato alla comprensione del "significato" del comportamento che il bambino manifesta e della risonanza emotiva che questo sollecita nelle rappresentazioni mentali genitoriali.

Può accadere che a seguito di traumi irrisolti pregressi, di fuorvianti aspettative rivolte al bambino o di percezioni distorte del bambino reale da parte dei genitori, questi ultimi non cercheranno di soddisfare i suoi reali bisogni ma distorceranno la sua immagine, affinché essa assuma particolari caratteristiche e connotazioni e si adegui a una propria immagine mentale (Zeanah, 2010); l'immagine del bambino "riparativo" o idealizzato prenderà il posto di quello reale, inducendo il genitore a una pressione intrusiva cui il bimbo reale si deve conformare. Il genitore non percepirà i reali bisogni, le capacità e le potenzialità del proprio bambino e la non avvenuta risoluzione, a seguito della mancata elaborazione, comporterà un permanere dei disturbi relazionali, con possibili conseguenze nefaste per lo sviluppo del bambino. Pattern di interazioni ripetitive di aspetti patologici del passato del genitore devono poter venire affrontate e "risolte" durante la "consultazione terapeutica", consentendo al genitore una maggiore capacità di assumere una prospettiva empatica verso quegli aspetti problematici del comportamento del bambino (Lieberman et al., 2003).

Oppenheim e Goldsmith (2010) rilevano come le relazioni di attaccamento, orientate a pattern di tipo sicuro, siano sostenute in particolare da una capacità di "insightfulness" dei genitori, intesa come una capacità intuitiva di "vedere il mondo" dal punto di vista del bambino. Questa capacità materna e genitoriale, di "tenere nella mente il mondo interno del bambino" (Koren Karie, Oppenheim, Goldsmith, 2010) è fondamentale per lo sviluppo emotivo dei bambini stessi, in circostanze normali, mentre un suo incremento è da considerarsi un obiettivo fondamentale quando la situazione è precaria e occorre un trattamento della relazione genitore-bambino (Fonagy et al., 1995; Silverman, Lieberman, 1999; Slade, 1999).

Si può operare una prospettiva di cambiamento a due livelli: favorire nel genitore un implementamento dell'insightfulness con cui egli potrà rappresentare con modalità più attinenti il bambino reale, $i$ suoi bisogni, le sue motivazioni, $i$ suoi desideri, che potrebbero anche cambiare a seconda del contesto, e contemporaneamente apprendere ad attivare uno "scanning" dei propri stati mentali, monitorando costantemente sia le proprie rappresentazioni del figlio, sia le proprie funzioni genitoriali (Slade, 2010). Un cambiamento del comportamento problematico genitoriale può avvenire attraverso una "riformulazione " delle rappresentazioni negative e distorte del bambino e della relazione, liberando i fantasmi dalla stanza del bambino. Si individuano così le modalità di attaccamento sicuro, come fattori protettivi per lo sviluppo stesso (Steele, Steele, 2008).

La capacità nel genitore di insightfulness e di comprensione delle motivazioni implicite del comportamento problematico dei figli è un fattore determinante per il cambiamento e il successo terapeutico (Fonagy et al., 1991; Lieberman, 1997; Zeanah, Benoit, 1995; Koren Karie, Oppenheim, Goldsmith, 2010) e una maggiore capacità genitoriale di insightfulness è correlata a una riduzione dei problemi comportamentali dei figli; al contrario, una mancanza di cambiamenti nelle rappresentazioni distorte che le madri hanno dei loro bambini è associata a un aumento delle difficoltà di comportamento nei bambini stessi (Oppenheim, Koren Karie, 2002). La capacità nel geni- 
tore di insightfulness favorisce lo sviluppo di una capacità di parenting adeguata a sostenere lo sviluppo del bambino e di una relazione di attaccamento sicuro (Oppenheim, Koren Karie, Sagi, 2001); la mancanza di insightfulness è un fattore di rischio che riduce i pattern di attaccamento sicuro e il senso di adeguatezza dei bambini (Oppenheim, Koren Karie, 2002). Questi concetti sono usati nella clinica: in situazioni di maltrattamento, di incuria e di traumi, è possibile ottenere un cambiamento nei bambini se si ottiene una tale "risoluzione".

Nei casi di adozione i bambini possono sperimentare nelle nuove famiglie modalità di vita più positive che consentono loro di poter "revisionare" i modelli insicuri di attaccamento (Steele et al., 2010): il supporto psicologico può sostenere i genitori adottivi nel processo di mantenimento di scambi emotivi positivi, aiutandoli a tollerare l'eventuale evitamento del bimbo, la sua e la propria aggressività, l'opposività e la provocazione, che sono tra le modalità comportamentali più frequenti nei bimbi che hanno subito maltrattamenti e abbandoni. I bambini traumatizzati in età precoce spesso hanno esperienze di fallimento e per loro risulta molto difficile poter attivare una regolazione emotivo-affettiva nelle interazioni, modalità che costituisce invece le basi per lo sviluppo di un sé stabile e organizzato, così come di un attaccamento sicuro. È necessario facilitare nel bambino la rievocazione di eventi passati positivi condivisi con i nuovi genitori, che possano contribuire a creare un nuovo legame di attaccamento. Il terapeuta può utilizzare le sedute in feedback, con i genitori per mettere in evidenza nuove modalità di comprensione del comportamento dei bambini adottati: attraverso un feedback in cui il terapeuta rivede insieme ai genitori alcune videosservazioni significative del trattamento, è possibile ritornare sui momenti cruciali della nuova relazione di attaccamento e "revisionare" le modalità interattive distorte, cercando percorsi più adeguati ai processi di sviluppo del bimbo.

Nei casi di affido le modalità che sembrano essere più positive e adeguate a un cambiamento nel bambino sono ciò che viene definito come "impegno" del genitore: la percezione della capacità del genitore affidatario di investire nella relazione con il bambino e il "coinvolgimento" emotivo nell'interazione con il figlio costituiscono una base affidabile e sicura per lo sviluppo del bambino stesso. Autori attuali stanno rivalutando le opportunità positive di queste due funzioni, "impegno" e "coinvolgimento", che avevano costituito una chiave di lettura importante nelle prime teorizzazioni di Bowlby, sviluppate purtroppo invece poco negli studi successivi (Dozier et al., 2010).

Nelle situazioni di affido può essere di basilare importanza, per questa popolazione di bambini, la percezione nel proprio genitore affidatario di quella che viene definita come "dedizione", cioè la capacità genitoriale di consolare e di calmare l'angoscia nel bambino, indicativa della qualità dell'attaccamento. La capacità di "dedizione" di un genitore è un aspetto di criticità nella relazione di affido, così come la speranza che il genitore mantenga una relazione costante e duratura.

Il poter disporre di un genitore "impegnato" nella relazione rappresenta una situazione non minacciosa e di maggiore sicurezza: il bambino che non ha avuto un caregiver impegnato è maggiormente vulnerabile alle minacce. Un nuovo genitore non coinvolto potrebbe non sacrificarsi nel giusto modo per proteggere il bambino dal pericolo, esponendolo a esperienze di allarme cronico. Il livello di impegno del 
caregiver è così un indice di previsione della durata dell'affido, della stabilità della relazione genitore-affidatario-bambino e degli esiti evolutivi delle cure affidatarie.

Nei casi di malattie croniche del bambino, è stato rilevato come elemento cruciale, per il senso di fiducia o sfiducia da parte dei genitori rispetto a come si rappresentano i problemi con il figlio, il modo in cui viene presentata loro la diagnosi (Oppenheim et al., 2010): la diagnosi di una patologia cronica può costituire una perdita metaforica del bimbo immaginato e desiderato, che verrebbe percepito come andato perduto per sempre, lasciando solo incognite e paure nei genitori per il suo sviluppo futuro. La possibilità di elaborare le emozioni suscitate dalla comunicazione della diagnosi, consente di revisionare le rappresentazioni non equilibrate del bambino e di sé, e di "risolvere" problematicità che potrebbero costituire nel tempo un fattore di ulteriore criticità nella cronicità della patologia stessa del bimbo, elevandone alla potenza gli eventuali effetti negativi e impedendo ai genitori di poter intravedere le acquisizioni evolutive del bimbo come segnali di miglioramento e di speranza per il suo sviluppo.

I bambini fanno affidamento sui caregiver per poter essere protetti dai pericoli e se subiscono traumi, se vengono maltrattati, abusati o abbandonati, viene gravemente compromessa la fiducia nella capacità delle loro figure di attaccamento (Bush, Lieberman, 2010). Le esperienze traumatiche minacciano la relazione di attaccamento. La capacità dei bambini di "recuperare" dopo essere stati sottoposti a relazioni violente è influenzata dalla qualità dei loro legami di attaccamento. Un bambino con relazioni di attaccamento sicure avrà rappresentazioni di sé e dell'altro positive e questo potrà favorire la sua fiducia nella possibilità di cercare e chiedere aiuto. I bambini con attaccamento insicuro sono più vulnerabili al trauma, in quanto privi o con scarse risorse interne emotive per far fronte al pericolo. Una terapia congiunta di intervento precoce consente al terapeuta di scandagliare la mente dei genitori, le loro esperienze traumatiche infantili che influenzano le funzioni genitoriali di accudimento e relazionali e poter così sostenere e potenziare gli aspetti sani dell'interazione.

I programmi di intervento precoce, per modificare il percorso evolutivo di genitori ad alto rischio e dei loro bambini (Powell et al., 2010), hanno l'obiettivo di aiutare i caregiver a rielaborare le proprie rappresentazioni interne, del bambino e del proprio sé, per svolgere una funzione di base sicura per il figlio: le rappresentazioni genitoriali organizzano i comportamenti di accudimento che questi mettono in atto nei confronti del proprio figlio e influenzano la sicurezza dell'attaccamento del bambino (Fonagy et al., 1991). La capacità riflessiva del genitore, favorita dall'intervento, può consentire la consapevolezza di quanto sia difficile, e in fondo doloroso, per un genitore dover dare al proprio figlio quello che nella sua infanzia non ha ricevuto, ma anche che potrà garantire protezione e sicurezza al suo bambino imparando a svolgere una funzione genitoriale attraverso l'aiuto terapeutico. Ovviamente gli interventi sono focalizzati prevalentemente sul genitore perché si ritiene che abbia maggiori capacità di attivare processi di cambiamento nella relazione.

Accanto e parallelamente alla psicoterapia genitore-bambino, altri progetti terapeutici vengono organizzati nel contesto scolastico, nonché in età prescolare, focalizzati a contrastare i modelli operativi interni negativi dei bambini identificati come insicuri nell'attaccamento con il caregiver principale, che tendono a stabilire con 
altri adulti e con i pari altrettante relazioni orientate alla sfiducia (Goldsmith, 2010). La psicoterapia con il genitore è focalizzata alle rappresentazioni negative che i genitori hanno del loro bambino e ha come obiettivo di stimolare lo sviluppo di maggiori capacità relazionali verso il figlio, in modo che si possa consentire la ristrutturazione del legame verso un pattern sicuro; il figlio viene inserito in uno specifico programma terapeutico entro cui può sperimentare interazioni positive con altri caregiver, che possano contrastare le rappresentazioni negative che egli ha degli adulti come punitivi e non disponibili.

Un interessante integrazione della teoria dell'attaccamento, entro una modalità di intervento secondo l'approccio psicoanalitico, è proposta dalla Slade (2010) e costituisce un ulteriore progresso tra i recenti contributi che provengono dalla ricerca e dalla clinica (Fonagy, 2000, 2001; Fonagy et al., 1995; Fonagy, Gergely, Jurist, 2002; Fonagy, Target, 1996, 1998; Hesse, Main, 2000; Main, 1996; Main, Hesse, 1992; Main Kaplan, Cassidy, 1985): la possibilità del terapeuta di "tenere nella sua mente" il genitore come paziente adulto considerando contemporaneamente il bambino che un tempo questi è stato, la sua infanzia, il suo coinvolgimento con gli oggetti interni primari. Ciò aiuta il terapeuta a dare un senso alle modalità che il paziente ha di regolare gli affetti e gli consente di "parlare" al paziente in un'area transizionale, in cui sia possibile costruire un senso condiviso alle sue esperienze, trovando modalità idonee a regolare le sue emozioni e permettendo di tradurre in parole i segnali di rabbia, paura, vergogna, senza voce e senza nome.

\subsection{Crescita e cambiamento genitoriale con il Modello Dinamico-Maturativo di Patricia Crittenden}

Patricia Crittenden (2009) nel testo Raising parents: Attachment, parenting and child safety affronta, attraverso il vertice teorico del suo Modello Dinamico Maturativo dell'attaccamento, le modalità di intervento con i genitori, aspetti che sono nucleo centrale del suo intervento nella seconda parte del presente volume. In particolare l'autrice affronta casi di popolazioni cliniche di genitori con disturbi emotivi che assumono comportamenti maltrattanti, abusanti o abbandonici nei confronti dei loro bambini e individua dei gradienti di distorsione delle informazioni su cui si basano questi comportamenti inadeguati, attivati in loro in contesti in cui un tempo il pericolo era presente e dunque indispensabili per la sopravvivenza e la protezione del sé del genitore (Crittenden, 1997), ma che risultano inadeguati nelle interazioni attuali con il proprio bambino.

Segnaliamo le indicazioni dell'autrice sulle prospettive di una crescita della funzione genitoriale in seguito a eventi fondamentali che possono accadere nella vita di un genitore e/o a seguito di interventi di sostegno alla genitorialità, o cambiamenti dovuti a terapie, con lo sviluppo di nuove capacità di lettura della realtà da parte del genitore.

Lo sviluppo infantile e la genitorialità si strutturano in base a esperienze di apprendimento interpersonale e a strategie protettive e di elaborazione mentale delle 
informazioni. Esiste una complementarità adattativa tra $\mathrm{i}$ comportamenti di cura dei genitori e i comportamenti di attaccamento del bambino: la funzione principale del parenting è quella di garantire al piccolo le condizioni di sicurezza fisica ed emotiva, necessarie alla sua sopravvivenza, attraverso il mantenimento della vicinanza con una figura protettiva. Da parte del bambino l'attaccamento si configura come processo attivo di adattamento che si organizza attraverso la messa a punto di strategie finalizzate al raggiungimento della sicurezza, le cui modalità variano in funzione sia delle caratteristiche ambientali, in primis quelle costituite dagli adulti, dalle quali il bimbo si ingegna a ottenere protezione e sicurezza, sia delle capacità cognitive, affettive e di organizzazione del comportamento che il bambino può raggiungere nelle diverse fasi dello sviluppo.

In queste ultime decadi è stata avviata una progressiva e interessante integrazione tra psicoanalisi infantile, interventi clinico-sperimentali derivati dall'Infant Research, teorie dell'attaccamento e neuroscienze, che ha prodotto una scoperta fondamentale: la maturazione neuropsichica, nel suo aspetto di sviluppo mentale e di strutturazione cerebrale, dipende dal tipo di relazione madre/feto/neonato/bambino e madre/padre/bambino. L'attenzione si è focalizzata sullo scambio emotivo-cognitivo della relazione genitore-bambino, in particolare sulla sua origine, formazione ed evoluzione e sull'importanza fondamentale, per la nascita del pensiero, delle prime relazioni affettive che il neonato stabilisce con la madre, con il padre e con chi svolge la funzione di caregiver: ciò può avvenire sia in senso positivo che patogenetico, a seconda del tipo di relazione. In psicologia clinica perinatale le ricerche sono concordi nell'evidenziare l'importanza della "qualità" della relazione primaria e di tutte le procedure psicologiche che possano diagnosticare le relazioni a rischio e proporre interventi atti a prevenire future anomalie psichiche e comunque a migliorare la maturazione neuropsichica dei bambini.

L'Infant Research ha studiato per via sperimentale gli scambi comunicativi primari nella relazione madre-bambino a partire dalle prime forme di imitazione e di intersoggettività e ha individuato nella madre le competenze di regolazione delle emozioni del bimbo, sia come etero regolazione che, in seguito, come autoregolazione. La regolazione emotiva viene identificata come la capacità del bimbo a mantenere la propria organizzazione comportamentale rispetto a stati di tensione e stress emotivo: egli apprende questa funzione nell'ambito delle interazioni primarie con la madre, la quale progressivamente gli insegna a regolare i suoi stati di tensione emozionale. Gli studi sulla sintonizzazione affettiva di Stern (1985) secondo il quale la madre, a partire dal secondo mese di vita, attraverso imitazioni delle espressioni del proprio bimbo riesce a sintonizzarsi con tali espressioni emotive rimandandogli emozioni e affetti in forme diverse, hanno messo in evidenza come per lo sviluppo delle strutture mentali della vita fetale e neonatale sia importante la sintonizzazione di un dialogo non verbale in primis tra la madre, il caregiver e il bimbo.

La capacità di riconoscere le proprie emozioni è un fattore determinante nella trasmissione delle prime modalità relazionali e di attaccamento tra genitore e figlio (Bowlby, 1988); tale capacità appare profondamente influenzata dal tipo di accessibilità emotiva che il genitore ha potuto a sua volta sperimentare nei confronti delle proprie figure di attaccamento, nel corso della propria storia infantile. 
Le modalità relazionali precoci tra il bambino e i suoi caregiver danno origine a stili individuali di regolazione affettiva che si ritrovano alla base dei successivi pattern di interazione; la modalità di regolazione emotiva che si attiva e si modula durante la relazione tra il bambino e il genitore viene interiorizzata e le esperienze affettive precoci influenzano e differenziano l'organizzazione dei legami di attaccamento, considerati relazioni di tipo emozionale, attraverso cui il bimbo impara peculiari stili di regolazione della propria emozione. Questi stili vengono considerati strategie adattative che il bambino utilizza per far fronte alle varie esperienze emotive. La disponibilità emotiva genitoriale a regolare gli stati affettivi del bimbo è fondamentale per il costituirsi del legame di attaccamento; la consapevolezza emotiva materna (Nichols, Gergely, Fonagy, 2001) è collegata alla qualità sicura dell'attaccamento del suo bimbo, l'attaccamento sicuro nel bimbo è correlato alla sua capacità di regolare il proprio stato emotivo, mentre l'attaccamento insicuro implica disfunzioni nelle capacità regolatorie del bimbo il cui genitore non è sufficientemente disponibile a modulare i suoi livelli di attivazione (Cassidy, 1994); tali modelli di attaccamento insicuri sono collegati a fallimentari tentativi di regolazione affettiva.

La teoria dell'attaccamento ha contribuito notevolmente a mettere in evidenza la caratteristica dei comportamenti non verbali materni negli scambi interattivi e l'importanza della sincronia e del contatto fisico ed emotivo nella costruzione dei pattern di attaccamento, consentendo un ampliamento di prospettiva di ricerca in diversi altri ambiti disciplinari.

In tale prospettiva si collocano i nuovi sviluppi evoluzionistici della teoria dell'attaccamento che hanno apportato interessanti contributi in questa direzione, con la costruzione di strumenti per la valutazione della relazione genitore-bambino (Crittenden, 1994) e di approfondimento delle modalità di valutazione dell'attaccamento adulto (Crittenden, 1999, 2008) nell'ottica di una maggiore comprensione degli sviluppi relazionali genitoriali attraverso l'influenza della trasmissione trasgenerazionale degli stili di attaccamento.

Attraverso processi di assessment per la valutazione della relazione genitorebambino e della funzione genitoriale, è possibile l'individuazione della qualità delle modalità di accudimento genitoriale che, a seconda delle esperienze genitoriali, potranno essere equilibrate e funzionali ai bisogni fisici ed emotivi dello sviluppo del bambino, oppure al contrario potranno essere più o meno condizionate dai bisogni del genitore stesso e quindi poco orientate al bimbo, che dovrà tuttavia in qualche modo corrispondere ad esse. È possibile effettuare una lettura e un'interpretazione dei dati con il DMM di Patricia Crittenden: sviluppo infantile e genitorialità sono connessi con la strutturazione, in base a strategie di apprendimento interpersonale, di strategie protettive e di elaborazione personale delle informazioni.

La lettura dei bisogni emotivi e affettivi del bimbo e delle capacità genitoriali di comprensione e di soddisfazione di tali bisogni è stata ampliata con la costruzione di strumenti e tecniche di videosservazione come il CARE-Index (Crittenden, 19792004), che consentono di rilevare dati empirici indicativi di fattori di rischio e di protezione nello sviluppo, mediante l'individuazione delle dimensioni del costrutto della "responsività sensibile" (Ainsworth, 1979), nel suo corrispettivo di sintonizzazione affettiva (Stern, 1985) attraverso un'integrazione con gli studi della scuola di Fo- 
nagy (Fonagy, Target, 2001), che hanno portato decisivi contributi agli sviluppi sugli stili di attaccamento e la trasmissione transgenerazionale di caratteristiche funzionali di base veicolate nelle vicende connesse all'accudimento-attaccamento.

Il riferimento alla "sensibilità" (Ainsworth, 1979) indica quella capacità genitoriale di soddisfare i bisogni non solo fisici, ma anche emotivi del bimbo con modalità e tempi adeguati al suo sviluppo: le madri modulano i loro ritmi a quelli di attivazione dei loro bambini e li aiutano a regolare il loro livello di attivazione. La Ainsworth rileva come i pattern di attaccamento sicuri e insicuri che il bimbo manifesta nei confronti del caregiver dipendono dalla sensibilità che questi ha avuto nei suoi riguardi nel corso del primo anno di vita. I pattern di attaccamento vengono considerati strategie adattative e difensive nei confronti della disponibilità emotiva delle figure affettive di riferimento che consentono al bambino di conservare il legame con il genitore. L'individuazione della "responsività sensibile" del genitore viene indicata come un fattore protettivo per lo sviluppo del bimbo e considerata indicatore importante nei processi di assessment precoce per la valutazione della relazione genitore-bambino.

Nei contesti di consultazione terapeutica vengono utilizzati strumenti per la valutazione dei legami di attaccamento, che vanno differenziati in funzione delle particolari modalità di espressione che essi assumono nel corso dello sviluppo

Mediante l'utilizzo di videosservazioni delle interazioni, attraverso la procedura del CARE-Index (Crittenden, 1979-2004) è possibile l'individuazione di configurazioni interattive precoci genitore-bambino che potranno costituire a un anno di età dei pattern di attaccamento.

Le videosservazioni delle interazioni genitore-bambino post-natali e dei primi mesi di vita del bimbo consentono di poter rilevare i "precursori" dell'attaccamento, attraverso una valutazione della responsività sensibile del genitore e della cooperazione del bambino.

I dati raccolti con il CARE-Index possono essere messi in relazione con i pattern di attaccamento, ricavati più avanti nello sviluppo del bimbo, all'anno di età, con la procedura della Strange Situation (Ainsworth et al., 1978), e la procedura del Preschool Assessment of Attachment PAA di Patricia Crittenden (Crittenden 19881994), che consente una più diretta valutazione del legame di attaccamento dai 18 mesi ai 5 anni.

Si può ottenere una maggiore comprensione delle modalità di accudimento e della funzione genitoriale attraverso una correlazione dei dati ricavati dalla somministrazione dei suddetti strumenti con quelli ottenuti dalla rilevazione dell'attaccamento nei genitori, con l'Adult Attachment Interview (George, Kaplan, Main, 1986, 1996; Crittenden, 1999, 2008), attraverso cui è possibile individuare le rappresentazioni mentali dell'attaccamento in soggetti adulti.

Presso la nostra sede, Dipartimento Materno Infantile, Università di Brescia, abbiamo in corso un progetto multidisciplinare osservazionale longitudinale, di prevenzione perinatale, pianificato secondo modalità metodologiche delle nuove prospettive evoluzionistiche della teoria dell'attaccamento e condotto attraverso processi e strumenti di valutazione precoce delle interazioni post-natali genitore-bambino, in relazione alla qualità delle funzioni genitoriali: tale studio si colloca in una prospet- 
tiva di screening preventivo generale della coppia genitoriale a partire dal periodo prenatale, in cui con gli strumenti di Patricia Crittenden e una lettura dei dati secondo il DMM cercheremo (capitolo 11) di individuare la popolazione a rischio e conseguentemente offrire un sostegno alla genitorialità ove si renda necessario.

\section{Bibliografia}

Ainsworth MDS (1979) Infant-mother attachment. American Psychology 34:932-937

Ainsworth MSD, Blehar MC, Waters E, Wall S (1978) Patterns of Attachment. Psychological Study of Strange Situation, Lawrence Erlbaum, Hillsdale, New Jersey

American Psychiatric Association (1994) Diagnostic and Statistical Manual of Mental Disorders. (4th edn), DSM-IV. Washington, DC

Ammaniti M (2001a) Manuale di psicopatologia dell'infanzia. Cortina, Milano

Ammaniti M (2001b) Disturbi post-traumatici da stress e abusi. In: Ammaniti M (a cura di) Manuale di psicopatologia dell'infanzia. Cortina, Milano

Ammaniti M (2001c) Quesiti e modelli nella psicopatologia infantile. In: Ammaniti M (a cura di) Manuale di psicopatologia dell'infanzia. Cortina, Milano

Ammaniti M (2001d) La diagnosi in età infantile. In: Ammaniti M (a cura di) Manuale di psicopatologia dell'infanzia. Cortina, Milano

Ammaniti M, Stern DN (a cura di) (1991) Rappresentazioni e narrazioni, Laterza, Bari

Baldoni F (2010) La prospettiva psicomatica. Il Mulino, Bologna

Bion WR (1970) Attenzione e interpretazione. Trad. it. Armando, Roma, 1973

Botvinick M, Jha AP et al (2005) Viewing facial expressions of pain engages cortical areas involved in the direct experience of pain. Neuroimaging 25:312-319

Bowlby J (1988) A secure base: parent-child attachment and health human development. Basic Books, New York, NY. Trad. it. Una base sicura. Cortina, Milano, 1989

Brazelton TB (1973) Neonatal Behavioral Assessment Scale (BNBAS). Brazelton, Trad.it Scala per la valutazione del comportamento del neonato. Casa Editrice Ambrosiana, Milano, 1977

Brazelton TB, Als H (1979) Four early stages in the development of mother-infant interaction. Psychoanalytic Study Child 34:349-369

Brazelton TB, Yogman M, Als H, Tronick E (1979) The infant as a focus in family reciprocity. In: Lewis M, Rosenblum M (a cura di) The child and its family. Plenum Press, New York

Brazelton TB(1992), Touchpoints: Your child's emotional and behavioural development. Perseus Publishing, Cambridge

Bush A, Lieberman AF (2010) Attaccamento e trauma. In: Goldsmith DF (a cura di) La teoria dell'attaccamento nel lavoro clinico con i bambini, Borla, Roma

Bydlowski M (1989) Desiderio di un bambino, desiderio di gravidanza, evoluzione delle pratiche della procreazione. Trad.it. Lebovici S. Weil-Halpern F, Psicopatologia della prima infanzia. Boringhieri, Torino

Bydlowski M, Raoul-Duval A (1978) Un avatar psychique méconnu: La névrose trumatique postobstétricale. Perspective Psychiatrique 68:321-328

Cammarella A, Lucarelli L, Vismara L (2001) L'osservazione del bambino nel contesto di sviluppo. In: Ammaniti M (a cura di) Manuale di psicopatologia dell'infanzia. Cortina, Milano

Candelori C, Mancone A(2001a).Genitorialità: situazioni a rischio e psicopatologiche. In: Ammaniti M (a cura di) Manuale di psicopatologia dell'infanzia. Cortina, Milano

Candelori C, Mancone A(2001b) Disturbi psicosamatici.In M Ammaniti, Manuale di psicopatologia dell'infanzia. Cortina, Milano

Carel A (1990) Le traumatisme à la naissance et les dysfonctionnements précoces au seine du groupe famille. In: Carel A et al (a cura di) Le nourrisson et sa famille. Cesura Lyon Edition, 99-112

Cassidy J (1994) Emotion regulation: influences of attachment relationship. In: Fox N (a cura di) 
The development of emotion regulation. Biological and behavioural consideration. Monographs of the Society for Research in Child Development 59:228-249

Cerutti R, Guidetti V (2007) Psicosomatica in età evolutiva. Il Pensiero Scientifico, Milano

Classificazione diagnostica (1994) Classificazione diagnostica 0-3 della salute mentale e dei disturbi di sviluppo nell'infanzia. Masson, Milano, 1997

Coates S, Cook C (2001) Disturbi dell'identità di genere. In: Ammaniti M (a cura di) Manuale di psicopatologia dell'infanzia. Cortina, Milano

Costa-Pinto F, Palermo-Neto J (2010) Neuroimmune interactions in stress. Neuroimmunomodulation 17:196-199

Cramer B (1990) Outcome evaluation in brief mother-infant psychotherapy: a preliminary report. Infant Mental Health Journal 11:278-300

Cramer B, Palacio-Espasa F (1993) Le psicoterapie madre-bambino. Trad. It. Masson, Milano, 1995

Cramer B, Stern DN (1988) La valutazione dei cambiamenti nella psicoterapia breve madre-bambino. In: Stern DN (a cura di) Le interazioni madre-bambino. Cortina, Milano

Crittenden PM (1979-2004) CARE-Index: Coding Manual. (Unpublished manuscript). Miami, FL

Crittenden PM (1988-1994) Preschool Assessment of Attachment Manual. Unpublished manuscript available from the author

Crittenden PM (1994) Nuove prospettive sull'attaccamento. Guerini e Associati, Milano

Crittenden PM (1997) Pericolo, sviluppo e adattamento. Trad.it. Masson, Milano

Crittenden PM (1999) Attaccamento in età adulta. L'approccio dinamico-maturativo all'Adult Attachment Interview, Cortina, Milano

Crittenden PM (2008) Il Modello Dinamico-Maturativo dell'Attaccamento. Cortina, Milano

Crittenden PM (2009) Raising parents: Attachment, parenting and child safety. Willan Publishing, UK

Crittenden PM (2011) Corpo o cervello? Yes! In: Crittenden PM (a cura di) DMM News 11 august 2011, 1-6

Dozier M, Grasso D, Lindhiem O, Lewis E (2010) La capacità di impegno del caregiver nelle cure affidatarie. In: Oppenheim D, Goldsmith DF (a cura di) La teoria dell'attaccamento nel lavoro clinico con i bambini, Borla, Roma

Eisenberger NI, Lieberman MD, Williams KD (2003) Does rejection hurt? An FMRI study of social exclusion. Science 302:290-292

Emde RN, Robinson J (1979) The first two months: recent research in developmental psychobiology and the changing view of the newborn, in Noshpitz JD, Basic Handbook of Child Psychiatry, Vol. I. Basic Books, New York

Engel GL (1977) The need for a new medical model: a challenge for biomedicine. Science 8:129136

Fabrizi A (2001) Disturbi affettivi. In Ammaniti M, Manuale di Psicopatologia dell'Infanzia. Cortina, Milano

Fivaz-Depeursinge E, Corboz-Warnery A, Frenck A (1992) L'approccio sistemico. In: Fava Viziello G, Stern DN (a cura di) Dalle cure materne all'interpretazione. Cortina, Milano

Fivaz-Depeursinge E, Corboz-Warnery A, Frenck A (1999) Il triangolo primario: le prime interazioni triadiche tra padre, madre e bambino. Cortina, Milano, 2000

Fivaz-Depeursinge E, Corboz-Warnery A, Carneiro C, Wasem V (2002) Coding manual for the GETCEF (Evaluation grid of the Lausanne Triadic Play). Third edition. Manoscritto non pubblicato. Centre d'Etude de la Famille (CEF), Lausanne

Fonagy P (2000) Attachment and borderline personality disorder. Journal of the American Psychoanalytic Association 48:1129-1146

Fonagy P (2001) Psicanalisi e teoria dell'attaccamento, Cortina, Milano

Fonagy P, Gergely G, Jurist EL, Target M (2002) Affect regulation, mentalization and the development of the self. New York; Other Press

Fonagy P, Steele M, Moran G, Steele M, Higgitt AC (1991) The capacity for understanding mental states: The reflective self in parent and child and its significance for security of attachment. Infant Mental Health Journal 13:200-216

Fonagy P, Steele M et al (1995) Attachment, the reflective self and borderline states: The predic- 
tive specificity of the Adult Attachment Interview and pathological emotional development. In: Goldberg S, Muir R, Kerr J (a cura di) Attachment theory: Social, developmental and clinical perspectives. Hillsdale, New York Analytic Press. Trad.it. Attaccamento, Sé Riflessivo e disturbi borderline. In: Riva Crugnola C (a cura di) La comunicazione affettiva tra il bambino e i suoi partner. Cortina, Milano, 1999

Fonagy P, Target M (1996) Playing with reality: I. Theory of mind and the normal development of psychic reality. International Journal of Psychoanalysis 77:217-233

Fonagy P, Target M (1998) Mentalization and the changing aims of child psychoanalysis. Psychoanalytic Dialogues 8:87-114

Fonagy P, Target M (2001) Attaccamento e funzione riflessiva. Cortina, Milano

Fraiberg SH (1980) Clinical Studies in Infant Mental Health. Basic Books, New York

Fraiberg SH (1999) Il sostegno allo sviluppo. Cortina, Milano

Fraiberg SH, Adelson E, Shapiro V (1975) I fantasmi nella stanza dei bambini. Un approccio psicoanalitico ai problemi posti da relazioni disturbate madre-bambino. Trad.it. In: Fraiberg SH (a cura di) Il sostegno allo sviluppo. Cortina, Milano, 1999

Furman E (1966) Parenthood as a Developmental Phase, Paper presented at the first scientific meeting of the Association for Child Psycoanalysis, Topeka, KS

Gaddini E (1981) Note sul problema mente-corpo. Rivista di Psicoanalisi 1:3-29

Gaddini E (1982) Early difensive fantasies and the psychoanalytical process. International Journal of Psychoanalysis 63:385-394

George C, Kaplan N, Main M (1986) The Adult Attachment Interview. Department of Psychology, University of California, Berkeley

George C, Kaplan N, Main M (1996) The Adult Attachment Interview. Department of Psychology, 3 ed., University of California, Berkeley

Goldsmith D (2010) Come contrastare I modelli operative interni negative dei bambini. In: Oppenheim D, Goldsmith DF (a cura di) La teoria dell'attaccamento nel lavoro clinico con i bambini, Borla, Roma

Golse B (1995) Le bébé, l’hôpital, les équipes soignantes et le "psys". In: Ben Soussan P (a cura di) Le bébé a l'hôpital. Syros, Paris

Golse B (2008) Essere-Bebé. Cortina, Milano

Grotstein JS (1986) The Psychology of Powerlessness: Disorders of Self-Regulation and Interactional regulation as a newer paradigm of psychopathology. Psychoanal Inq 6:93-118

Gunnar M, Herrera A, Hostinar C (2009) Stress and early brain development. Montreal, QC

Hesse E, Main M (2000) Disorganized infant, child and adult attachment. Journal of American Psychoanalytic Association 48:1097-1129

Hofer MA (1983a) The mother-infant interaction as a regulator of infant physiology and behaviour. In L Rosenblum, H Moltz (a cura di), Symbiosis in Parent-Offspring Interactions. Plenum, New York

Hofer MA (1983b) On the relationship between attachment and separation processes in infancy. In: Plutchik R, Kellermann H (a cura di) Emotion: theory, research and experience. Academic Press, New York

Hofer MA (1984) Relationship as regulators: A psychobiologic perspective on bereavement. Psychosomatic Medicine 46:183-197

Hoffman IZ (1994) Dialectic thinking and therapeutic action in the psychoanalytic process. Psychoanalytic Quarterly 63:187-218

ICD-10-CM (1992) International Classification of Diseases, Tenth Revision, Clinical Modification. (WHO) World Health Organization

Imbasciati A (2006a) Constructing a Mind. A new base for Psychoanalytic theory. Brunner-Routledge, London

Imbasciati A (2006b) Il Sistema Protomentale. LED, Milano

Imbasciati A (2008a) Le cure materne: matrice dello sviluppo del futuro individuo. Nascere XXXV:4-14

Imbasciati A., Margiotta M (2008b) Psicologia Clinica, manuale per la formazione degli Operatori della Salute.Piccin, Padova 
Imbasciati A, Calorio D (1981) Il Protomentale. Boringhieri, Torino

Imbasciati A, Cena L (2008) Le cure materne in una prospettiva transgenerazionale. Nascere XXXVI:4-15

Imbasciati A, Cena L (2010) I bambini e i loro caregiver. Borla, Roma

Imbasciati A, Dabrassi F, Cena L (2011) Psicologia Clinica Perinatale per lo sviluppo dei futuri individui. Espress, Torino

Ionescu S, Jacquette MJ, Lhote C (1997) Les méchanismes de défense, théorie et clinique. Nathan, Paris

Kaplan B, Letourneau N, Giesbrecht G, Campbell T, Kooistra L, Pop V (2010) Effects of maternal stress and mood on infant stress reactivity. Alberta Centre for Child, Family, and Community Research, 434

Kernberg OF (1992) Aggression in Personality Disorders and Perversions. Yale Univ Press, New Haven. Trad. it. Aggressività, disturbi della personalità e perversioni. Cortina, Milano, 1993

Klein M (1932) La psicoanalisi dei bambini. Martinelli, Firenze, 1969

Koren-Karie N, Oppenheim D, Goldsmith DF (2010) Tenere nella mente il mondo interno del bambino. In: Oppenheim D, Goldsmith DF (a cura di) La teoria dell'attaccamento nel lavoro clinico con i bambini, Borla, Roma

Kowallis GH (1995) Psychosomatic Disorders. In: Lionells M, Fiscalini J, Mann CH, Stern DN (eds) Handbook of Interpersonal Psychoanalysis. The Analytic Press, New York

Kozyrskyj A, Mai X, McGrath P, HayGlass K, Becker A, MacNeil B (2008) Continued exposure to maternal distress in early life is associated with an increased risk of childhood asthma. American Journal of Respiratory and Critical Care Medicine 177:142-147

Kreisler L (1981) Clinica psicosomatica del bambino. Cortina, Milano

Kreisler L (1985) Conduites alimentaires déviantes du bbé. A. L'anorexie mentale.B. La rumination ou mérycisme. C. Les vomissements psychogènes. In: Lebovici S, Diatkine R, Soulé M (a cura di) Nouveau traité de psychiatrie de l'enfant et de l'adolescent. PUF, Quadrige, Paris, 1999

Kreisler L (1992) Les origines de la depression essentielle. La linee depressive. Revue française de psychosomatique 9:15-33

Kreisler L, Cramer B (1981) Les bases cliniques de la psychiatrie du nourrisson. Psychiatrie de l'enfant XXIV:223-263

Kreisler L, Fain M, Soulé M (1974) Il bambino e il suo corpo. Astrolabio, Roma, 1976

Lebovici S (1980) L'Expérience du psychanaliste chez l'enfant et chez l'adulte devant le modèle de la névrose infantile et de la névrose de transfert. Revue Française de psychoanalise 44:5-6

Lebovici S (1983) Il neonato, la madre e lo psicoanalista. Le interazioni precoci. Trad. it. Borla, Roma, 1988

Lebovici S (1989) I legami intergenerazionali. Le interazioni fantasmatiche. Trad. it. Lebovici S, Weil-Halpern F (a cura di) Psicopatologia della prima infanzia. Boringhieri, Torino, 1994

Lebovici S (1992) L'homme dans le bébé. Eshel, Paris

Lebovici S, Weil-Halpern F (1989), Psychopathologie du bébé. PUF, Paris. Trad. it. Psicopatologia della prima infanzia. Boringhieri, Torino

Leifer M (1977) Psychological changes accompanying pregnancy and motherhood. Genetical Psychology Monography 95:55-96

Letourneau N, Watson,B. Duffett-Leger L, Hegadoren K (2011) Maternal-infant interactions are related to cortisol levels in mothers and infants affected by postpartum depression

Letourneau NL, Fedick CB, Willms JD, Dennis CL, Hegadoren K, Stewart MJ (2006) Longitudinal study of postpartum depression, maternal-child relationships and children's behaviour to 8 years of age. In: Devore D (a cura di) Parent-child relations: New research, 45-63. Nova Science Publishers, New York, NY

Lichtenberg JD (1983) La psicoanalisi e l'osservazione del bambino. Trad. it. Astrolabio, Roma, 1988

Lieberman AF (1997) Toddlers'internalization of maternal attribution as a factor in quality of attachment. In: Atkinson L, Zucker KJ (a cura di) Attachment and psychopathology, Guilford Press, New York

Lieberman AF Compton NC, Vah Horn P, Ghosh Ippen C (2003) Losing a parent to death in the 
early years. Zero to three Press, Washington. Tr. it. Il lutto infantile. Il Mulino, Bologna

Lieberman AF, Pawl JH (1993) Infant-parent psychoterapy. In: Zeanah CH (ed) Handbook of infant mental health, Guilford, New York, 1993

Lucarelli L (2001) Disturbi dell'alimentazione. In: Ammaniti M (a cura di) Manuale di psicopatologia dell'infanzia. Cortina, Milano

Lucarelli L, Vismara L (2001) Disturbi del sonno. In: Ammaniti M (a cura di) Manuale di psicopatologia dell'infanzia. Cortina, Milano

Mahler M, Pine F, Bergman A (1975) La nascita psicologica del bambino. Tr.it. Boringhieri, Torino

Main M (1996) Una visione d'insieme sulla teoria dell'attaccamento. Tr.it. In Carli L (a cura di) Dalla diade alla famiglia. Cortina, Milano, 1999

Main M, Hesse, E (1992) Attaccamento disorganizzato/disorientato nell'infanzia e stati mentali dissociati dei genitori. In: Ammaniti M, Stern DN (a cura di) Attaccamento e psicoanalisi. Laterza, Bari

Main M, Kaplan N, Cassidy J (1985) Security in infancy childhood and adulthood: a move to the level of representation. Tr.it. Riva Crugnola (a cura di) Lo sviluppo affettivo del bambino. Cortina, Milano, 1993

Mancia M (1989) Vie prénatale et naissance du soi. In: Pasini W et al (a cura di) Le foetus et son entourage. Médicine et hygiène. Genéve, 5-18

Manzano J, Palacio-Espasa F, Zilkha N (2001) Scenari della genitorialità. Cortina, Milano

Marcelli D, Paget A, Blossier P (1996) les origine du travail de penser entre mére et bébé. In Psychiatrie de l'enfant XXXIX :540

Marty P, de M'uzan M (1963) La pensée opératoire. Revue Francaise de Psychoanalyse 27:13451356

McDonough SC (1992) L'aiuto all'interazione: una tecnica per il trattamento dei disturbi relazionali precoci. In: Stern DN, Fava Vizziello G (a cura di) Dalle cure materne all'interpretazione. Cortina, Milano

McDonought SC (2006) Guida all'interazione: promuovere e sostenere la relazione di Caregiving. In: Sameroff AJ, McDonough SC, Rosenblum KL (a cura di) Il trattamento clinico della relazione genitore-bambino. Il Mulino, Bologna

McHale JP (2010) La sfida della cogenitorialità. Cortina, Milano

Missonnier S. (2005) La consultazione terapeutica perinatale. Cortina, Milano

Muratori F, Cosenza A, Parrini B (2001) Disturbi multisistemici dello sviluppo.In M Ammaniti, Manuale di psicopatologia dell'infanzia. Cortina, Milano

Nichols K, Gergely G, Fonagy P (2001) Experimental protocols for investigating relationships among mother-infant interaction, affect regulation, physiological markers of stress responsiveness, and attachment. Bulletin of The Menninger Clinic 3:371-379. Guilford Publications, New York, NY

Oppenheim D, Dolev S et al (2010) La risoluzione genitoriale della diagnosi del bambino e la relazione genitore-figlio. In: Oppenheim D, Goldsmith DF (a cura di) La teoria dell'attaccamento nel lavoro clinico con i bambini, Borla, Roma

Oppenheim D, Goldsmith DF (2010) La teoria dell'attaccamento nel lavoro clinico con i bambini, Borla, Roma

Oppenheim D, Koren-Karie N (2002) Mothers'Insightfulness Regarding their Children's Internal Worlds: The capacity underlying secure child-mother relationships. Infant Mental Health Journal 23:593-605

Oppenheim D, Koren-Karie N, Sagi A (2001) Mothers' empathic understanding of their preschoolers' internal experience: Relations with early attachment. International Journal of Behavioral Development 25:16-26

Palacio-Espasa F, Manzano J (1982) La consultation thérapeutique des très jeunes enfants et leur mères. Psichiatrie de l'enfant XXI:25

Palacio-Espasa F, Cramer B (1989) Psychotherapie de la relation mère-enfant. Revue de Médicine Psychosomatique 19:59-70

Papousek H, Papousek N (1975) Cognitive aspects of preverbal social interaction between human infants and adults. In: O'Connor (a cura di) Parent-infant interaction. Elsevier, Amsterdam 
PDM Task Force (2006) Manuale Diagnostico Psciodinamico. Trad. it.(a cura di) Del Corno, Lingiardi. Cortina, Milano 2008

Perard-Cupa D, Valdes L, Abadie I, Pineiro M, Lazartigues A (1992) Bébé imaginé et interactions précoces. In Devenir 4:47-60

Perucon M (1997) Le rituel et son pouvoir de liaison. Perspectives Psychanalitiques. In: Perucon $\mathrm{M}$, Rites de vie, rites de mort. Puff, Paris

Powell B, Cooper G, Hoffman K, Marvin R (2010) Il progetto del circolo sicurezza. In: Oppenheim D, Goldsmith DF (a cura di) La teoria dell'attaccamento nel lavoro clinico con i bambini, Borla, Roma

Presaghi F, Cerutti R (2007) Metodologie e strumenti di valutazione nella psicosomatica in età evolutiva. In: Cerutti R, Guidetti V (a cura di) Psicosomatica in età evolutiva. Il Pensiero Scientifico, Milano

Sameroff AJ, Emde RN (1989) I disturbi delle relazioni nella prima infanzia. Trad. it. Boringhieri, Torino, 1991

Sameroff AJ, McDonough SC, Rosenblum KL (2004) Treating parent-infant relationship problems. The Guilford Press, New York. Trad. it, Il trattamento clinico della relazione genitore-bambino. Il Mulino, Bologna

Sander LW (1975) Infant and caretaking environment. In Anthony EJ, Explorations in Child Psychiatry, Plenum, New York

Sander LW (1987) Awareness of inner experience: a system perspective on self-regulatory process in early development, Child Abuse and Neglect 11:339-346

Seligman S (1994) Applying Psychoanalysis in an Unconventional Context: Adapting Infant-Parent Psychotherapy to a Changing Population. In The Psychoanalytic Study of the Child 49:481500

Shonkoff J (2004) Science, policy, and the young developing child: Closing the gap between what we know and what we do. Ounce of Prevention Fund, 1-12

Silverman R, Lieberman A (1999) Negative maternal attributions, projective identification and the intergenerational transmission of violent relational patterns. Psychoanalytic Dialogues 9:161186

Slade A (1999) Representation, symbolization and affect regulation in the concomitant treatment of a mother and a child. Attachment theory and Child psychoterapy. Psychoanalitich Inquiry 19:797-830

Slade A (2010) Madre disorganizzata, bambino disorganizzato. In: Oppenheim D, Goldsmith DF (a cura di) La teoria dell'attaccamento nel lavoro clinico con i bambini, Borla, Roma

Solano L (2001) Tra Mente e Corpo: come si costruisce la Salute. Cortina, Milano

Speranza AM (2001a) Disturbi della regolazione. In: Ammaniti M (a cura di) Manuale di psicopatologia dell'infanzia. Cortina, Milano

Speranza AM (2001b) Disturbi da deficit di attenzione, iperattività e disturbi della condotta. In: Ammaniti M (a cura di) Manuale di psicopatologia dell'infanzia. Cortina, Milano

Spitz R (1946) Anaclitic depression. In The Psychoanalytic Study of the Child 2:313-342

Steele H, Steele M (2008) Ten clinical uses of the Adult Attachment Interview. In: Steele H, Steele M (a cura di) Clinical applications of the Adult Attachment Interview. Guilford Press, New York

Steele M, Hodges J, Kaniuk J, Steele H, D'Agostino D, Blom I, Hillmann S, Henderson K (2010) Interventi con I bambini maltrattati e con le loro famiglie adottive. In: Oppenheim D, Goldsmith DF (a cura di) La teoria dell'attaccamento nel lavoro clinico con i bambini, Borla, Roma

Stern DN (1971) Una microanalisi dell'interazione madre-bambino. In: DN Stern (a cura di), Le interazioni madre-bambino. Cortina, Milano

Stern DN (1977) Le prime relazioni sociali: il bambino e la madre. Trad. it. Sovera Multimedia, Roma, 1989

Stern DN (1983) The early development of schemes of self., of other, and of various experiences of "self with others", In: Kaplan S, Lichtenberg J (a cura di), Rejections on self psychology. International Universities Press, New York

Stern DN (1985) The Interpersonal World of the Infant, Basic Books, New York. Trad.it. Il mondo interpersonale del bambino. Boringhieri, Torino, 1987 
Stern DN (1989) La rappresentazione dei modelli di relazione. In: Sameroff A, Emde RN (a cura di), I disturbi delle relazioni nella prima infanzia. Boringhieri, Torino, 1991

Stern DN (1991) Maternal representations: a clinical and subjective phenomenological view. Infant Mental Health Journal 12:173-185

Stern DN (1992) Aspetti fondamentali delle terapie genitore-bambino: I fattori comuni nei diversi approcci. In: Fava-Vizziello G, Stern DN (a cura di) Dalle cure materne all'interpretazione. Cortina, Milano

Stern DN (1995) La costellazione materna. Boringhieri, Torino

Stern DN (2006) La costellazione materna: approcci terapeutici ai problemi relazionali precoci. In Sameroff AJ, McDonough SC, Rosenblum KL (a cura di), Il trattamento clinico della relazione genitore-bambino, Il Mulino, Bologna

Stern-Bruschweiler N, Stern DN (1989) A model for conceptualizing the role of the mother's representational world in various mother-infant therapies. Infant Mental Health Journal 10:142156

Stoleru S (1989) La genitorializzazione e i suoi disturbi. Trad. it. Lebovici S, Weil-Hallpern F, Psicopatologia della prima infanzia. Boringhieri, Torino

Stoleru S, Morales M, Grinshpoun MF (1985) De l'enfant fantasmatique de la grossesse à l'interaction mère-nourrisson. In Psychiatrie de l'enfant 2:441-484

Tailor GJ (1987) Psychosomatic medicine and contemporary psychoanalysis. International Universities Press, Madison, WI

Tailor GJ, Bagby RM, Parker JDA (2000) Disorders of Affect Regulation: Alexithymia in Medical and Psychiatric Illness (Paperback edition 1999). Cambridge University Press. In Disturbi della regolazione affettiva. Fioriti, Roma

Trevarthen C, Hubley P (1978) Secondary intersubjectivity: Confidence, confiding and acts of meaning in the first year. In: Lock A (a cura di), Action, gesture, and symbol: The emergence of language (183-227). Cambridge University Press, New York

Trevarthen C (1980), The foundations of intersubjectivity: Development of interpersonal and cooperative understanding in infancy. In: Olson D (a cura di), The social foundations of language and though: Essays in honor of JS Bruner. Northon, New York.

Vallino D (2004) La consultazione con il bambino e i suoi genitori. Rivista di Psicoanalisi XLVIII:2

Vallino D (2007) La Consultazione partecipata: figlie genitori nella stanza dell'analisi. In: Uno spazio per i genitori. Quaderni di Psicoterapia infantile. Borla, Roma

Vallino D (2009) Fare psicoanalisi con genitori e bambini. Borla, Roma

Winnicott DW (1958) Dalla pediatria alla psicoanalisi. Martinelli, Firenze, 1975

Winnicott DW (1965) Sviluppo affettivo e ambiente. Armando, Roma, 1970

Winnicott DW (1966) L'inizio dell'individuo. Trad. it. I bambini e le loro madri. Cortina, Milano, 1987

Winnicott DW (1971) Colloqui terapeutici con il bambino. Trad. It. Armando, Roma, 1974

Winnicott DW (1977) The Piggle. An Account of the Psychoanalytic Treatment of a Little Girl. Hogarth Press, London and Inst of Psa, Madison

Winnicott DW (1989) I bambini e le loro madri. Armando, Roma

Zeanah CH (2010) Come costruire una formulazione della relazione madre-bambino. In: Oppenheim D, Goldsmith DF (a cura di) La teoria dell'attaccamento nel lavoro clinico con i bambini, Borla, Roma

Zeanah H., Benoit D (1995) Clinical applications of a parent perception interview in infant mental health. Child and Adolescent Psychiatric Clinics of North America, Infant Psychiatry 4:539554 